Department of Economics, Hitotsubashi University

Discussion Paper Series \#2016-07

Confidence Sets for the Break Date in Cointegrating Regressions

Eiji Kurozumi Anton Skrobotov

September 2016 


\title{
Confidence Sets for the Break Date in Cointegrating Regressions ${ }^{1}$
}

\author{
Eiji Kurozumi \\ Department of Economics \\ Hitotsubashi University
}

\author{
Anton Skrobotov \\ Institute of Applied Economic Studies \\ Russian Presidential Academy of National \\ Economy and Public Administration
}

September 28, 2016

\begin{abstract}
In this paper, we propose constructing confidence sets for a break date in cointegrating regressions by inverting a test for the break location, which is obtained by maximizing the weighted average of power. It is found that the limiting distribution of the test depends on the number of I(1) regressors whose coefficients sustain structural change and the number of I(1) regressors whose coefficients are fixed throughout the sample. By Monte Carlo simulations, we then show that compared with a confidence interval developed by using the existing method based on the limiting distribution of the break point estimator under the assumption of the shrinking shift, the confidence set proposed in the present paper has a more accurate coverage rate, while the length of the confidence set is comparable. By using the method developed in this paper, we then investigate the cointegrating regressions of Russian macroeconomic variables with oil prices with a break.
\end{abstract}

JEL classification: $\mathrm{C} 12, \mathrm{C} 21$

Keywords: Confidence interval, structural change, cointegration, Russian economy, oil price

\footnotetext{
${ }^{1}$ We are grateful to Andrey Polbin for helpful comments and valuable suggestions. All errors are our responsibility. Kurozumi's research was partly supported by JSPS KAKENHI Grant Number 16K03594.
} 


\section{Introduction}

This paper proposes constructing a confidence set for the change point in cointegrating regressions. Cointegration has long been an important concept for investigating the long-run relationships among macroeconomic variables. To capture the long-run relationship, data over relatively long time-frames are often used in such an investigation. In this case, we should take into account that the economic structure may change during the sample period. For example, Campos, Ericsson, and Hendry (1996) investigate the effect of structural change on cointegration tests and Gregory and Hansen (1996a, b) propose tests for the null hypothesis of no cointegration that are robust to the existence of structural change, while tests for the null hypothesis of cointegration with a structural break are proposed by Carrion-i-Silvestre and Sansó (2006) and Arai and Kurozumi (2007). On the contrary, tests for structural change in the framework of cointegrating regressions have been proposed by Bai, Lumsdaine, and Stock (1998, BLS hereafter) and Kejriwal and Perron (2010). By using the tests presented in the literature in addition to the careful inspection of original data and economic events, we may find cointegrating relations with structural change. In this case, a statistical inference about the change point can be made by using the method proposed by BLS in the case of a single break, while multiple breaks were investigated by Kejriwal and Perron (2008a).

In the case of regressions using stationary variables, the break point is estimated by minimizing the sum of the squared residuals or by using the quasi-maximum likelihood method, while the confidence interval is constructed by using the limiting distribution of the break point estimator, as suggested by Bai (1997) and Bai and Perron (1998). In this case, the crucial assumption made for the construction of the confidence interval is that the magnitude of the structural break shrinks to 0 at a rate slower than $1 / \sqrt{T}$, as also assumed in BLS and Kejriwal and Perron (2008a). However, as demonstrated by Elliott and Müller (2007) and Chang and Perron (2015), a confidence interval based on the limiting distribution of the break point estimator tends to be too liberal when the magnitude of the break is not so large. Instead of using the limiting distribution of the change point estimator, Elliott and Müller (2007) propose constructing a confidence interval by inverting the test for the break location, which helps control the coverage rate. However, the drawback of their method, 
as pointed out by Chang and Perron (2015), is that the confidence interval tends to be too wide. Indeed, it covers most of the sample period in some cases, thereby offering no useful information in practice. To overcome this drawback, Yamamoto (2016) pays attention to the estimation of the long-run variance for the construction of the test for the break location and proposes estimating it by taking the estimated break point into account, while Kurozumi and Yamamoto (2015) consider a similar method to Elliott and Müller (2007) but propose inverting the sup-type, average-type, and exponential-type tests for the break location, which can be obtained by maximizing the average power of a test. By Monte Carlo simulations, it is shown that these methods can better control the coverage rate and that the length of the confidence set becomes close to or smaller than that based on Bai (1997). On the contrary, Eo and Morley (2015) investigate a confidence set based on the likelihood ratio, while Harvey and Leybourne (2015) propose constructing a confidence set for the date of a break in level and trend that is valid for both $\mathrm{I}(0)$ and $\mathrm{I}(1)$ processes. Further, Kurozumi (2016) extends the method of Kurozumi and Yamamoto (2015) to linear regression models with non-homogeneous regressors, particularly with a linear trend.

As in the above case of stationary regressions, controlling the coverage rate of the confidence interval of the break date in the case of cointegrating regressions may be difficult based on the methods of BLS and Kejriwal and Perron (2008a). Indeed, the simulation results reported in these papers are not necessarily satisfactory. Therefore, in this paper, we propose constructing a confidence set by inverting the test for the break location in cointegrating regressions. In this case, while the basic structure of the test is the same as in the case of regressions with stationary regressors, the limiting distribution becomes different. We derive the asymptotic distribution of the test depending on whether a linear trend is included in the regressions and/or the coefficient associated with the $\mathrm{I}(1)$ regressors sustain a structural change. As in the case of stationary regressions, the critical values depend on the location of the break fraction under the null hypothesis and it is inconvenient to tabulate them for all the permissible break fractions in practical analysis. Instead, as in Kurozumi and Yamamoto (2015), we conduct response surface regressions and propose obtaining the critical values of the test for the break location in a simple formula. By Monte Carlo simulations, we show that our method can control the coverage rate of the confidence set better than BLS, while 
the size of the confidence set based on our method is comparable to that of BLS.

The rest of the paper is organized as follows. Section 2 introduces the model and assumptions. We separately consider models for whether a linear trend is included in the cointegrating regressions and/or the coefficients of the I(1) regressors change after the break point. In Section 3, we propose constructing the confidence set by inverting the test for the break location. The finite sample property of our method is investigated by Monte Carlo simulations in Section 4, and we apply the method developed in this paper to a Russian economic analysis in Section 5. Section 6 offers concluding remarks. The mathematical proofs are relegated to Appendix A.

\section{Model and Assumptions}

Let us consider the following linear model:

$$
y_{t}=w_{b, t}^{\prime} \beta_{b}+w_{b, t}\left(\lambda_{0}\right)^{\prime} \delta_{b}+w_{f, t}^{\prime} \beta_{f}+e_{t}
$$

for $t=1, \cdots, T$, where $w_{b, t}, w_{b, t}\left(\lambda_{0}\right)$, and $w_{f, t}$ are $p_{b^{-}}, p_{b^{-}}$, and $p_{f}$-dimensional regressors, respectively, $w_{b, t}\left(\lambda_{0}\right)=1\left(t>\left[\lambda_{0} T\right]\right) w_{b, t}$ with $1(\cdot)$ being an indicator function, $\lambda_{0}$ is a true break fraction, $[a]$ denotes the largest integer less than $a, e_{t}$ is an error term, and $\beta_{b}, \delta_{b}$, and $\beta_{f}$ are $p_{b^{-}}, p_{b^{-}}$, and $p_{f}$-dimensional unknown coefficients, respectively. Since this paper aims to construct a confidence set for the break date, we assume that a one-time structural change occurred in the sample period and the true break date is denoted as $T_{0}=\left[\lambda_{0} T\right]$. Note that the coefficient associated with $w_{b, t}$ sustains a structural change from $\beta_{b}$ to $\beta_{b}+\delta_{b}$, while $\beta_{f}$ is fixed throughout the sample period.

Since we consider a cointegrating regression model, the regressors $w_{b, t}$ and/or $w_{f, t}$ include I(1) variables, which are given by

$$
\begin{aligned}
& z_{b, t}=z_{b, t-1}+u_{b, t}^{z} \\
& z_{f, t}=z_{f, t-1}+u_{f, t}^{z},
\end{aligned}
$$

where $z_{b, t}$ and $z_{f, t}$ are $p_{b}^{z}$ - and $p_{f}^{z}$-dimensional, respectively. In the following analysis, we mainly proceed without a drift in the $\mathrm{I}(1)$ variables even when $w_{b, t}$ includes a linear trend. 
As discussed in Remark 2, the main result in regressions with the $\mathrm{I}(1)$ variables with a drift remains the same by slightly modifying the test statistics in the next section.

In general, the error terms $u_{b, t}^{z}$ and $u_{f, t}^{z}$ are correlated with the regression error $e_{t}$ in (1) and this correlation will become a nuisance parameter in our test. To eliminate the correlation, we include as regressors the leads-and-lags of the first differences of the $\mathrm{I}(1)$ variables in model (1), as suggested by Phillips and Loretan (1991), Saikkonen (1991), and Stock and Watson (1993). Such a model becomes

$$
y_{t}=w_{b, t}^{\prime} \beta_{b}+w_{b, t}\left(\lambda_{0}\right)^{\prime} \delta_{b}+w_{f, t}^{\prime} \beta_{f}+\sum_{j=-l}^{l} \pi_{b, j}^{\prime} \Delta z_{b, t-j}+\sum_{j=-l}^{l} \pi_{f, j}^{\prime} \Delta z_{f, t-j}+u_{t},
$$

where $\Delta$ denotes the first differencing operator and both the leads-and-lags lengths are $l$ for notational convenience, but they can be different as in, for example, Hayakawa and Kurozumi (2008) and Choi and Kurozumi (2012). In the following, we assume that $u_{t}$ are uncorrelated with $u_{b, t-j}^{z}$ and $u_{f, t-j}^{z}$ for all $j$.

Remark 1 In general, the leads-and-lags lengths are infinite and thus if we truncate them at some point $l$, then the error term $u_{t}$ includes an additional noise caused by the truncation. However, this noise can be negligible asymptotically under additional assumptions, as proven by Saikkonen (1991), Arai and Kurozumi (2007), and Kejriwal and Perron (2008b) among others. We thus consider the finite leads-and-lags as in (2) only because we would like to avoid complicating the proof further; the results obtained in the paper also hold in the general infinite leads-and-lags case.

For notational convenience, we bundle up the additional stationary regressors $\Delta z_{b, t-j}$ and $\Delta z_{f, t-j}$ and denote them as a $p_{f}^{x}$-dimensional vector $x_{f, t}$. Since $x_{f, t}$ consists of the first differences of the $\mathrm{I}(1)$ regressors, we assume $E\left[x_{f, t}\right]=0$ throughout the paper. In addition, because the coefficient associated with $x_{f, t}$ is fixed throughout the sample, we absorb $x_{f, t}$ into $w_{f, t}$ and then the model can be simply expressed as

$$
\begin{aligned}
y_{t} & =w_{b, t}^{\prime} \beta_{b}+w_{b, t}\left(\lambda_{0}\right)^{\prime} \delta_{b}+w_{f, t}^{\prime} \beta_{f}+u_{t} \\
& =w_{t}\left(\lambda_{0}\right)^{\prime} \beta+u_{t},
\end{aligned}
$$


where $w_{f, t}$ includes $x_{f, t}$ and $w_{t}\left(\lambda_{0}\right)=\left[w_{b, t}^{\prime}, w_{b, t}\left(\lambda_{0}\right)^{\prime}, w_{f, t}^{\prime}\right]^{\prime}$. Model (3) can be expressed in vector form as

$$
\begin{aligned}
y & =W_{b} \beta_{b}+W_{b}\left(\lambda_{0}\right) \delta_{b}+W_{f} \beta_{f}+u \\
& =W\left(\lambda_{0}\right) \beta+u
\end{aligned}
$$

where $y=\left[y_{1}, \cdots, y_{T}\right]^{\prime}, W_{b}=\left[w_{b, 1}, \cdots, w_{b, T}\right]^{\prime}, W_{b}\left(\lambda_{0}\right)=\left[0, \cdots, 0, w_{b, T_{0}+1}, \cdots, w_{b, T}\right]^{\prime}, W_{f}=$ $\left[w_{f, 1}, \cdots, w_{f, T}\right]^{\prime}, W\left(\lambda_{0}\right)=\left[W_{b}, W_{b}\left(\lambda_{0}\right), W_{f}\right]$, and $u=\left[u_{1}, \cdots, u_{T}\right]^{\prime}$.

As a specification of the regressors $w_{b, t}$ and $w_{f, t}$, we consider the following commonly used models in practical analysis:

Model I-a : A constant and the I(1) regressors are included in the cointegrating relation and all the coefficients except for those associated with the first differences of the I(1) regressors sustain a break. That is, $w_{b, t}=\left[1, z_{b, t}^{\prime}\right]^{\prime}, w_{f, t}=x_{f, t}, \beta_{b}=\left[\beta_{b, c}, \beta_{b, z}^{\prime}\right]^{\prime}$, $\delta_{b}=\left[\delta_{b, c}, \delta_{b, z}^{\prime}\right]^{\prime}$, and $\beta_{f}=\beta_{f, x}:$

$$
y_{t}=\beta_{b, c}+z_{b, t}^{\prime} \beta_{b, z}+1\left(t>\left[\lambda_{0} T\right]\right)\left(\delta_{b, c}+z_{b, t}^{\prime} \delta_{b, z}\right)+x_{f, t}^{\prime} \beta_{f, x}+u_{t} .
$$

Model I-b : A constant and the I(1) regressors are included in the cointegrating relation and only a constant term sustains a break. That is, $w_{b, t}=1, w_{f, t}=\left[z_{f, t}^{\prime}, x_{f, t}^{\prime}\right]^{\prime}, \beta_{b}=\beta_{b, c}$, $\delta_{b}=\delta_{b, c}$, and $\beta_{f}=\left[\beta_{f, z}^{\prime}, \beta_{f, x}^{\prime}\right]^{\prime}$ :

$$
y_{t}=\beta_{b, c}+1\left(t>\left[\lambda_{0} T\right]\right) \delta_{b, c}+z_{f, t}^{\prime} \beta_{f, z}+x_{f, t}^{\prime} \beta_{f, x}+u_{t} .
$$

Model I-c : A constant and the I(1) regressors are included in the cointegrating relation and some of the coefficients associated with the I(1) regressors are fixed throughout the sample. That is, $w_{b, t}=\left[1, z_{b, t}^{\prime}\right]^{\prime}, w_{f, t}=\left[z_{f, t}^{\prime}, x_{f, t}^{\prime}\right]^{\prime}, \beta_{b}=\left[\beta_{b, c}, \beta_{b, z}^{\prime}\right]^{\prime}, \delta_{b}=\left[\delta_{b, c}, \delta_{b, z}^{\prime}\right]^{\prime}$, and $\beta_{f}=\left[\beta_{f, z}^{\prime}, \beta_{f, x}^{\prime}\right]^{\prime}:$

$$
y_{t}=\beta_{b, c}+z_{b, t}^{\prime} \beta_{b, z}+1\left(t>\left[\lambda_{0} T\right]\right)\left(\delta_{b, c}+z_{b, t}^{\prime} \delta_{b, z}\right)+z_{f, t}^{\prime} \beta_{f, z}+x_{f, t}^{\prime} \beta_{f, x}+u_{t} .
$$

Model II-a : A constant, a linear trend, and the I(1) regressors are included in the cointegrating relation and all the coefficients except for those associated with the first 
differences of the $\mathrm{I}(1)$ regressors sustain a break. That is, $w_{b, t}=\left[1, t, z_{b, t}^{\prime}\right]^{\prime}, w_{f, t}=x_{f, t}$, $\beta_{b}=\left[\beta_{b, c}, \beta_{b, \tau}, \beta_{b, z}^{\prime}\right]^{\prime}, \delta_{b}=\left[\delta_{b, c}, \delta_{b, \tau}, \delta_{b, z}^{\prime}\right]^{\prime}$, and $\beta_{f}=\beta_{f, x}$ :

$$
y_{t}=\beta_{b, c}+\beta_{b, \tau} t+z_{b, t}^{\prime} \beta_{b, z}+1\left(t>\left[\lambda_{0} T\right]\right)\left(\delta_{b, c}+\delta_{b, \tau} t+z_{b, t}^{\prime} \delta_{b, z}\right)+x_{f, t}^{\prime} \beta_{f, x}+u_{t} .
$$

Model II-b : A constant, a linear trend, and the I(1) regressors are included in the cointegrating relation and only a constant term and a linear trend sustain a break. That is, $w_{b, t}=[1, t], w_{f, t}=\left[z_{f, t}^{\prime}, x_{f, t}^{\prime}\right]^{\prime}, \beta_{b}=\left[\beta_{b, c}, \beta_{b, \tau}\right]^{\prime}, \delta_{b}=\left[\delta_{b, c}, \delta_{b, \tau}\right]^{\prime}$, and $\beta_{f}=\left[\beta_{f, z}^{\prime}, \beta_{f, x}^{\prime}\right]^{\prime}:$

$$
y_{t}=\beta_{b, c}+\beta_{b, \tau} t+1\left(t>\left[\lambda_{0} T\right]\right)\left(\delta_{b, c}+\delta_{b, \tau} t\right)+z_{f, t}^{\prime} \beta_{f, z}+x_{f, t}^{\prime} \beta_{f, x}+u_{t} .
$$

Model II-c : A constant, a linear trend, and the I(1) regressors are included in the cointegrating relation and some of the coefficients associated with the I(1) regressors are fixed throughout the sample. That is, $w_{b, t}=\left[1, t, z_{b, t}^{\prime}\right]^{\prime}, w_{f, t}=\left[z_{f, t}^{\prime}, x_{f, t}^{\prime}\right]^{\prime}, \beta_{b}=$ $\left[\beta_{b, c}, \beta_{b, \tau}, \beta_{b, z}^{\prime}\right]^{\prime}, \delta_{b}=\left[\delta_{b, c}, \delta_{b, \tau}, \delta_{b, z}^{\prime}\right]^{\prime}$, and $\beta_{f}=\left[\beta_{f, z}^{\prime}, \beta_{f, x}^{\prime}\right]^{\prime}:$

$y_{t}=\beta_{b, c}+\beta_{b, \tau} t+z_{b, t}^{\prime} \beta_{b, z}+1\left(t>\left[\lambda_{0} T\right]\right)\left(\delta_{b, c}+\delta_{b, \tau} t+z_{b, t}^{\prime} \delta_{b, z}\right)+z_{f, t}^{\prime} \beta_{f, z}+x_{f, t}^{\prime} \beta_{f, x}+u_{t}$.

Let $z_{u, t}=\sum_{j=1}^{t} u_{j}$ and $z_{t}=\left[z_{b, t}^{\prime}, z_{f, t}^{\prime}, z_{u, t}\right]^{\prime}=\left[z_{1, t}^{\prime}, z_{u, t}\right]^{\prime}$, where $z_{1, t}=\left[z_{b, t}^{\prime}, z_{f, t}^{\prime}\right]^{\prime}$. For model (3), we make the following assumption.

Assumption 1 (a) The following weak convergences hold:

$$
\begin{gathered}
\frac{1}{\sqrt{T}} z_{[r T]} \Rightarrow B^{*}(r) \quad \text { for } \quad 0 \leq r \leq 1, \\
\frac{1}{T} \sum_{t=1}^{[r T]} z_{1, t} u_{t} \Rightarrow \int_{0}^{r} B_{1}^{*}(r) d B_{u}^{*}(r)^{\prime}, \quad 0 \leq r \leq 1
\end{gathered}
$$

where $B^{*}(r)=\left[B_{1}^{*}(r)^{\prime}, B_{u}^{*}(r)\right]^{\prime}=\left[B_{b}^{*}(r)^{\prime}, B_{f}^{*}(r)^{\prime}, B_{u}^{*}(r)\right]^{\prime}$ is a $\left(p_{b}^{z}+p_{f}^{z}+1\right)$-dimensional Brownian motion with $E\left[B^{*}(s) B^{*}(t)^{\prime}\right]=\min (s, t) \Omega=\min (s, t) \operatorname{diag}\left\{\Omega_{11}, \omega_{u u}\right\}$ for $0 \leq s, t \leq 1$, $\Omega_{11}>0, \omega_{u u}>0$, and $\Rightarrow$ signifies the weak convergence of the associated probability measures.

(b) $x_{f, t}$ is a stationary process with $E\left[x_{f, t}\right]=0, E\left[x_{f, t} u_{t-j}\right]=0$ for all $j$, and the following relations hold:

$$
\frac{1}{T} \sum_{t=1}^{T} x_{f, t} x_{f, t}^{\prime} \stackrel{p}{\longrightarrow} \Sigma_{x}>0,
$$




$$
\begin{gathered}
\frac{1}{\sqrt{T}} \sum_{t=1}^{T} x_{f, t} u_{t}=O_{p}(1), \\
\frac{1}{T} \sum_{t=1}^{[r T]} z_{t} x_{t}^{\prime}=O_{p}(1), \quad 0 \leq r \leq 1,
\end{gathered}
$$

where $\stackrel{p}{\longrightarrow}$ signifies convergence in probability.

Assumption 1(a) implies that the functional central limit theorem holds for the I(1) regressors and the partial sum process based on $u_{t}$. Since the leads-and-lags of the I(1) regressors are included in model $(3), u_{t}$ is supposed to be uncorrelated with all the leadsand-lags of $\Delta z_{b, t}$ and $\Delta z_{f, t}$, meaning that the so-called second-order bias does not appear in (6). Assumption 1(b) requires that the innovations driving the $\mathrm{I}(1)$ regressors are zero-mean stationary processes and that the well-known mild moment conditions also hold, as in (8) and (9).

\section{Confidence Set for the Break Date}

In this section, we consider constructing a confidence set for the break date by inverting the test for the location of the break point. For the unknown break point, we test for

$$
H_{N}: T_{0}=T_{1} \quad \text { vs. } \quad H_{A}: T_{0}=T_{2}
$$

with the significance level $\alpha$, and if the null hypothesis is accepted, then we include $T_{1}$ in the confidence set; otherwise we exclude $T_{1}$ from the confidence set. By conducting this test for all the permissible break points, we obtain a confidence set for the break date with the confidence level $1-\alpha$. Note that testing problem (10) can be expressed by using the break fraction as

$$
H_{N}: \lambda_{0}=\lambda_{1} \quad \text { vs. } \quad H_{A}: \lambda_{0}=\lambda_{2}
$$

where $\lambda_{1}=T_{1} / T$ and $\lambda_{2}=T_{2} / T$.

In this procedure, the confidence set becomes smaller as a test becomes more powerful and, therefore, we should construct a test as powerful as possible. However, it is not difficult to see that there exists no uniformly most powerful test for testing problem (10). Instead, 
following the literature, we consider constructing a test that maximizes the weighted average of power.

We first note that we cannot directly estimate (3) by using $w_{b, t}\left(\lambda_{0}\right)$ because $w_{b, t}\left(\lambda_{0}\right)$ depends on the unknown break fraction $\lambda_{0}$. Since the testing problem is given by (10), we consider estimating the model under the null hypothesis and construct the test statistic. Let $w_{b, t}\left(\lambda_{1}\right)=1\left(t>\left[\lambda_{1} T\right]\right) w_{b, t}$ and $r_{t}\left(\lambda_{0}, \lambda_{1}\right)=w_{b, t}\left(\lambda_{0}\right)-w_{b, t}\left(\lambda_{1}\right)$. Then, model (3) can be expressed as

$$
\begin{aligned}
y_{t} & =w_{b, t}^{\prime} \beta_{b}+w_{b, t}\left(\lambda_{0}\right)^{\prime} \delta+w_{f, t}^{\prime} \beta_{f}+u_{t} \\
& =w_{b, t}^{\prime} \beta_{b}+w_{b, t}\left(\lambda_{1}\right)^{\prime} \delta+w_{f, t}^{\prime} \beta_{f}+u_{t}+r_{t}\left(\lambda_{0}, \lambda_{1}\right)^{\prime} \delta \\
& =w_{t}\left(\lambda_{1}\right)^{\prime} \beta+u_{t}\left(\lambda_{0}, \lambda_{1}\right),
\end{aligned}
$$

where $w_{t}\left(\lambda_{1}\right)=\left[w_{b, t}^{\prime}, w_{b, t}\left(\lambda_{1}\right)^{\prime}, w_{f, t}^{\prime}\right]^{\prime}$ and $u_{t}\left(\lambda_{0}, \lambda_{1}\right)=u_{t}+r_{t}\left(\lambda_{0}, \lambda_{1}\right)^{\prime} \delta$. In vector form, this becomes

$$
\begin{aligned}
y & =W_{b} \beta_{b}+W_{b}\left(\lambda_{0}\right) \delta+W_{f} \beta_{f}+u \\
& =W_{b} \beta_{b}+W_{b}\left(\lambda_{1}\right) \delta+W_{f} \beta_{f}+u+R\left(\lambda_{0}, \lambda_{1}\right) \delta \\
& =W\left(\lambda_{1}\right) \beta+u\left(\lambda_{0}, \lambda_{1}\right),
\end{aligned}
$$

where $W_{b}\left(\lambda_{1}\right)$ and $W\left(\lambda_{1}\right)$ are defined similarly to $W_{b}\left(\lambda_{0}\right)$ and $W\left(\lambda_{0}\right)$ in (4) with $\lambda_{0}$ replaced by $\lambda_{1}, R\left(\lambda_{0}, \lambda_{1}\right)=W_{b}\left(\lambda_{0}\right)-W_{b}\left(\lambda_{1}\right)$, and $u\left(\lambda_{0}, \lambda_{1}\right)=u+R\left(\lambda_{0}, \lambda_{1}\right) \delta$. From expression (13), we restrict our attention to a class of tests invariant to the group of transformations given by $y \rightarrow y+W\left(\lambda_{1}\right) b$ and $\beta \rightarrow \beta+b$, where $b$ is a $\left(2 p_{b}+p_{f}\right)$-dimensional vector. Following Andrews and Ploberger (1994), Elliott and Müller (2007), and Kurozumi and Yamamoto (2015) among others, we consider maximizing the weighted average of power over the magnitude of the break $\delta$ and the location of the break under the alternative $\lambda_{2}$, which is given by

$$
\iint P\left(\varphi \text { rejects } H_{N} \mid \delta, \lambda_{2}\right) d Q_{\lambda_{2}}(\delta) d J\left(\lambda_{2}\right)
$$

where $\varphi$ is a test for (11) with the significance level $\alpha$ and $Q_{\lambda_{2}}(\delta)$ and $J\left(\lambda_{2}\right)$ are non-negative measures on $\mathbb{R}^{p_{b}}$ and $(0,1)$, respectively. Typically, these weighting functions are chosen so that the asymptotic distribution of the test statistic becomes free of the nuisance parameters. 
In the case of cointegrating regressions, we set

$$
Q_{\lambda_{2}}(\delta) \sim N\left(0, \frac{c \sigma^{2}}{T} \Sigma_{\delta}^{-1}\right) \quad \text { where } \quad \Sigma_{\delta}=\frac{1}{T} R\left(\lambda_{2}, \lambda_{1}\right)^{\prime} M_{w_{1}} R\left(\lambda_{2}, \lambda_{1}\right)
$$

with $M_{w_{1}}=I_{T}-W\left(\lambda_{1}\right)\left(W\left(\lambda_{1}\right)^{\prime} W\left(\lambda_{1}\right)\right)^{-1} W\left(\lambda_{1}\right)^{\prime}$ and $R\left(\lambda_{2}, \lambda_{1}\right)=W_{b}\left(\lambda_{2}\right)-W_{b}\left(\lambda_{1}\right)$, while $J\left(\lambda_{2}\right)$ is a uniform measure on $\Lambda_{\varepsilon}=\left\{\lambda_{2}: \varepsilon \leq \lambda_{2}<\lambda_{1}-\varepsilon, \lambda_{1}+\varepsilon<\lambda_{2} \leq 1-\varepsilon\right\}$, in which both the end points and the neighborhood of $\lambda_{1}$ are excluded to avoid the explosive behavior of the test statistic. Then, in the same manner as Andrews and Ploberger (1994), Elliott and Müller (2007), and Kurozumi and Yamamoto (2015), under the additional assumption that $\left\{u_{t}\right\}$ is a sequence of i.i.d. normal random variables and $\left\{u_{t}\right\}$ is independent of $\left\{w_{b, t}\right\}$ and $\left\{w_{f, t}\right\}$, the test that maximizes the averaged power given by (14) rejects the null hypothesis when

$$
\begin{aligned}
\widetilde{L R}_{T}\left(\lambda_{1}\right)= & \int_{\lambda_{2} \in \Lambda_{\varepsilon}}(1+c)^{-p_{\beta} / 2} \exp \left\{\frac{c}{2(1+c) \sigma^{2}}\right. \\
& \left.y^{\prime} M_{w_{1}} R\left(\lambda_{2}, \lambda_{1}\right)\left(R\left(\lambda_{2}, \lambda_{1}\right)^{\prime} M_{w_{1}} R\left(\lambda_{2}, \lambda_{1}\right)\right)^{-1} R\left(\lambda_{2}, \lambda_{1}\right)^{\prime} M_{w_{1}} y\right\} d \lambda_{2}>a
\end{aligned}
$$

for some value $a$. Note that the assumption of normality is used only for the derivation of the test statistic, and we do not assume normality in the remainder of the paper.

We first derive the limiting distribution of $\widetilde{L R}_{T}\left(\lambda_{1}\right)$ for each model. Let us partition the long-run variance $\Omega_{11}$ in Assumption 1 (a) into $\Omega_{i j}$ for $i, j=b$ and $f$. Define

$$
\begin{gathered}
B_{b}(r)=\Omega_{b b}^{-1 / 2} B_{b}^{*}(r), \quad B_{f}(r)=\Omega_{f f}^{-1 / 2} B_{f}^{*}(r), \quad B_{u}(r)=\omega_{u u}^{-1 / 2} B_{u}^{*}(r), \\
B_{f \cdot b}(r)=\Omega_{f \cdot b}^{-1 / 2}\left(B_{f}^{*}(r)-\Omega_{f b} \Omega_{b b}^{-1} B_{b}^{*}(r)\right)
\end{gathered}
$$

where $\Omega_{f \cdot b}=\Omega_{f f}-\Omega_{f b} \Omega_{b b}^{-1} \Omega_{b f}$. Note that they are all standard Brownian motions and that $B_{b}(r), B_{f \cdot b}(r)$, and $B_{u}(r)$ are independent of each other.

Theorem 1 Suppose Assumption 1 holds. Then, under the null hypothesis $H_{N}$, we have

$$
\begin{aligned}
\widetilde{L R}_{T} & \Rightarrow \widetilde{L R}\left(\lambda_{1}\right) \\
& \equiv \int_{\lambda_{2} \in \Lambda_{\epsilon}}(1+c)^{-p_{\beta} / 2} \exp \left(\frac{c}{2(1+c)} G\left(\lambda_{1}, \lambda_{2}\right)^{\prime} H\left(\lambda_{1}, \lambda_{2}\right)^{-1} G\left(\lambda_{1}, \lambda_{2}\right)\right) d \lambda_{2}
\end{aligned}
$$


where (a) for Models I- $a$ and II- $a$,

$$
\begin{aligned}
& G\left(\lambda_{1}, \lambda_{2}\right)= \\
& \left\{\begin{array}{l}
\int_{\lambda_{2}}^{\lambda_{1}} \tilde{B}_{b}(r) d B_{u}(r)-\int_{\lambda_{2}}^{\lambda_{1}} \tilde{B}_{b}(r) \tilde{B}_{b}(r)^{\prime} d r\left(\int_{0}^{\lambda_{1}} \tilde{B}_{b}(r) \tilde{B}_{b}(r)^{\prime} d r\right)^{-1} \int_{0}^{\lambda_{1}} \tilde{B}_{b}(r) d B_{u}(r) \quad: \quad \lambda_{2}<\lambda_{1} \\
-\int_{\lambda_{1}}^{\lambda_{2}} \tilde{B}_{b}(r) d B_{u}(r)-\int_{\lambda_{1}}^{\lambda_{2}} \tilde{B}_{b}(r) \tilde{B}_{b}(r)^{\prime} d r\left(\int_{\lambda_{1}}^{1} \tilde{B}_{b}(r) \tilde{B}_{b}(r)^{\prime} d r\right)^{-1} \int_{\lambda_{1}}^{1} \tilde{B}_{b}(r) d B_{u}(r) \quad: \quad \lambda_{2}>\lambda_{1}
\end{array}\right.
\end{aligned}
$$$$
H\left(\lambda_{1}, \lambda_{2}\right)=
$$$$
\left\{\begin{array}{l}
\int_{\lambda_{2}}^{\lambda_{1}} \tilde{B}_{b}(r) \tilde{B}_{b}(r)^{\prime} d r-\int_{\lambda_{2}}^{\lambda_{1}} \tilde{B}_{b}(r) \tilde{B}_{b}(r)^{\prime} d r\left(\int_{0}^{\lambda_{1}} \tilde{B}_{b}(r) \tilde{B}_{b}(r)^{\prime} d r\right)^{-1} \int_{\lambda_{2}}^{\lambda_{1}} \tilde{B}_{b}(r) \tilde{B}_{b}(r) d r \quad: \quad \lambda_{2}<\lambda_{1} \\
\int_{\lambda_{1}}^{\lambda_{2}} \tilde{B}_{b}(r) \tilde{B}_{b}(r)^{\prime} d r-\int_{\lambda_{1}}^{\lambda_{2}} \tilde{B}_{b}(r) \tilde{B}_{b}(r)^{\prime} d r\left(\int_{\lambda_{1}}^{1} \tilde{B}_{b}(r) \tilde{B}_{b}(r)^{\prime} d r\right)^{-1} \int_{\lambda_{1}}^{\lambda_{2}} \tilde{B}_{b}(r) \tilde{B}_{b}(r) d r \quad: \quad \lambda_{2}>\lambda_{1}
\end{array}\right.
$$

with $\tilde{B}_{b}(r)=\left[1, B_{b}(r)^{\prime}\right]^{\prime}$ for Model I- $a$ and $\tilde{B}_{b}(r)=\left[1, r, B_{b}(r)^{\prime}\right]^{\prime}$ for Model II- $a$,

(b) for Models I-b and II-b,

$$
\begin{gathered}
G\left(\lambda_{1}, \lambda_{2}\right)=\left\{\begin{array}{lll}
\int_{\lambda_{2}}^{\lambda_{1}} \tilde{B}_{b}(r) d B_{u}(r)-J_{1}\left(\lambda_{1}, \lambda_{2}\right)^{\prime} K\left(\lambda_{1}\right)^{-1} L\left(\lambda_{1}\right) & : & \lambda_{2}<\lambda_{1} \\
-\int_{\lambda_{1}}^{\lambda_{2}} \tilde{B}_{b}(r) d B_{u}(r)-J_{2}\left(\lambda_{1}, \lambda_{2}\right)^{\prime} K\left(\lambda_{1}\right)^{-1} L\left(\lambda_{1}\right) & : & \lambda_{2}>\lambda_{1}
\end{array}\right. \\
H\left(\lambda_{1}, \lambda_{2}\right)=\left\{\begin{array}{lll}
\int_{\lambda_{2}}^{\lambda_{1}} \tilde{B}_{b}(r) \tilde{B}_{b}(r)^{\prime} d r-J_{1}\left(\lambda_{1}, \lambda_{2}\right)^{\prime} K\left(\lambda_{1}\right)^{-1} J_{1}\left(\lambda_{1}, \lambda_{2}\right) & : \quad \lambda_{2}<\lambda_{1} \\
\int_{\lambda_{1}}^{\lambda_{2}} \tilde{B}_{b}(r) \tilde{B}_{b}(r)^{\prime} d r-J_{2}\left(\lambda_{1}, \lambda_{2}\right)^{\prime} K\left(\lambda_{1}\right)^{-1} J_{2}\left(\lambda_{1}, \lambda_{2}\right) & : \quad \lambda_{2}>\lambda_{1}
\end{array}\right.
\end{gathered}
$$

with

$$
\begin{gathered}
J_{1}\left(\lambda_{1}, \lambda_{2}\right)=\left[\int_{\lambda_{2}}^{\lambda_{1}} \tilde{B}_{b}(r) \tilde{B}_{b}(r)^{\prime} d r, 0, \int_{\lambda_{2}}^{\lambda_{1}} \tilde{B}_{b}(r) B_{f}(r)^{\prime} d r\right] \\
J_{2}\left(\lambda_{1}, \lambda_{2}\right)=\left[0,-\int_{\lambda_{1}}^{\lambda_{2}} \tilde{B}_{b}(r) \tilde{B}_{b}(r)^{\prime} d r,-\int_{\lambda_{1}}^{\lambda_{2}} \tilde{B}_{b}(r) B_{f}(r)^{\prime} d r\right] \\
K\left(\lambda_{1}\right)=\left[\begin{array}{ccc}
\int_{0}^{\lambda_{1}} \tilde{B}_{b}(r) \tilde{B}_{b}(r)^{\prime} d r & 0 & \int_{0}^{\lambda_{1}} \tilde{B}_{b}(r) B_{f}(r)^{\prime} d r \\
0 & \int_{\lambda_{1}}^{1} \tilde{B}_{b}(r) \tilde{B}_{b}(r)^{\prime} d r & \int_{\lambda_{1}}^{1} \tilde{B}_{b}(r) B_{f}(r)^{\prime} d r \\
\int_{0}^{\lambda_{1}} B_{f}(r) \tilde{B}_{b}(r)^{\prime} d r & \int_{\lambda_{1}}^{1} B_{f}(r) \tilde{B}_{b}(r)^{\prime} d r & \int_{0}^{1} B_{f}(r) B_{f}(r)^{\prime} d r
\end{array}\right] \\
L\left(\lambda_{1}\right)=\left[\left(\int_{0}^{\lambda_{1}} \tilde{B}_{b}(r) d B_{u}(r)\right)^{\prime},\left(\int_{\lambda_{1}}^{1} \tilde{B}_{b}(r) d B_{u}(r)\right)^{\prime},\left(\int_{0}^{1} B_{f}(r) d B_{u}(r)\right)^{\prime}\right]^{\prime}
\end{gathered}
$$

with $\tilde{B}_{b}(r)=1$ for Model I-b and $\tilde{B}_{b}(r)=[1, r]^{\prime}$ for Model II-b,

(c) for Models I-c and II-c, G( $\left.\lambda_{1}, \lambda_{2}\right), H\left(\lambda_{1}, \lambda_{2}\right), J_{1}\left(\lambda_{1}, \lambda_{2}\right), J_{2}\left(\lambda_{1}, \lambda_{2}\right), K\left(\lambda_{1}\right)$, and $L\left(\lambda_{1}\right)$ are defined as in Case (b) with $B_{f}(r)$ replaced by $B_{f \cdot b}(r)$ with $\tilde{B}(r)=\left[1, B_{b}(r)^{\prime}\right]^{\prime}$ for Model I-c and $\tilde{B}(r)=\left[1, r, B_{b}(r)^{\prime}\right]^{\prime}$ for Model II-c. 
Remark 2 When the I(1) regressors have a drift term, we typically consider including a linear trend in the regressions. Because a constant and a linear trend (with a break) are included as regressors in Models II- a to II-c, the test statistic $\widetilde{L R}_{T}\left(\lambda_{1}\right)$ is invariant to the drift term of the I(1) regressors if we replace $\Delta z_{b, t-j}$ and $\Delta z_{f, t-j}$ in $x_{f, t}$ with the demeaned versions, $\Delta z_{b, t-j}-\overline{\Delta z_{b}}$ and $z_{f, t-j}-\overline{\Delta z_{f}}$, respectively, to ensure that $E\left[x_{f, t}\right]=0$. In this case, we obtain the same result as given in Theorem 1. Note that when the I(1) regressors have a drift term in Models I-a to I-c, the limiting distributions would be different from those given in Theorem 1, but we do not pursue these cases in this paper because it is natural and practically more relevant to include a linear trend as in Models II-a to II-c when the I(1) variables have a drift.

As we can see from Theorem 1, the limiting distributions become simpler for the models without the I(1) regressors with fixed coefficients because in those models, the regressor $w_{b, t}$, whose coefficients sustain structural change, becomes asymptotically orthogonal to the other regressors $w_{f, t}$ with fixed coefficients. On the contrary, when the $\mathrm{I}(1)$ regressors are included in $w_{f, t}, w_{b, t}$ is correlated with $w_{f, t}$ even in the limit and thus the limiting distribution of the test statistic depends on $p_{f}^{z}$, the number of $\mathrm{I}(1)$ regressors with fixed coefficients.

Note that the limiting distribution depends on the localizing parameter $c$ and we have to deal with $c$ to implement the test. Following Andrews (1993) and Andrews and Ploberger (1994), we consider the sup-type, average-type, and exponential-type tests. Their limiting expressions are given by

$$
\begin{aligned}
\sup -L R\left(\lambda_{1}\right) & =\sup _{\lambda_{2} \in \Lambda_{\epsilon}} G\left(\lambda_{1}, \lambda_{2}\right)^{\prime} H\left(\lambda_{1}, \lambda_{2}\right)^{-1} G\left(\lambda_{1}, \lambda_{2}\right) \\
\operatorname{avg}-L R\left(\lambda_{1}\right) & =\lim _{c \rightarrow 0} \frac{2}{c}\left(\widetilde{L R}\left(\lambda_{1}\right)-1\right) \\
& =\int_{\lambda_{2} \in \Lambda_{\epsilon}} G\left(\lambda_{1}, \lambda_{2}\right)^{\prime} H\left(\lambda_{1}, \lambda_{2}\right)^{-1} G\left(\lambda_{1}, \lambda_{2}\right) d \lambda_{2}, \\
\exp -L R(\lambda) & =\lim _{c \rightarrow 0} \log (1+c)^{p_{\beta} / 2} \widetilde{L R}\left(\lambda_{1}\right) \\
& =\log \int_{\lambda_{2} \in \Lambda_{\epsilon}} \exp \left(\frac{1}{2} G\left(\lambda_{1}, \lambda_{2}\right)^{\prime} H\left(\lambda_{1}, \lambda_{2}\right)^{-1} G\left(\lambda_{1}, \lambda_{2}\right)\right) d \lambda_{2} .
\end{aligned}
$$

The critical values of these distributions depend on $\lambda_{1}$ and it is inconvenient to tabulate them for all the permissible break fractions $\lambda_{1}$. Instead, we first calculate critical values 
for $\lambda_{1}$ ranging from 0.1 to 0.9 in increments of 0.01 , for the given significance level, over $\Lambda_{\varepsilon}=\left\{\lambda_{2}: \varepsilon \leq \lambda_{2}<\lambda_{1}-\varepsilon, \lambda_{1}+\varepsilon<\lambda_{2} \leq 1-\varepsilon\right\}$ with $\varepsilon=0.05$, where a standard Brownian motion is approximated by the normalized partial sums of standard normal random variables. ${ }^{2}$ Next, we conduct the response surface regression by using the obtained critical values for each case as a function of $\lambda_{1}$. The regression form is given by

$$
\text { critical value }=a_{0}+a_{-1} \frac{1}{\ell+1}+a_{1} \ell+a_{2} \ell^{2}+a_{3} \ell^{3}
$$

where $\ell=\left|\lambda_{1}-0.5\right|$. The obtained coefficients are summarized in Appendix B. ${ }^{3}$ Note that the regression coefficients $a_{0}$ to $a_{3}$ depend on the test statistics, significance level, number of I(1) regressors whose coefficients sustain a break, and number of I(1) regressors whose coefficients are fixed throughout the sample period.

From the limiting expressions (15)-(17), the corresponding finite sample versions, which can be implemented in practical analysis, are given by

$$
\begin{aligned}
\sup -L R_{T}\left(T_{1}\right) & =\max _{T_{2} \in \mathcal{T}_{\epsilon}} F_{T_{2}}\left(T_{1}\right), \\
\operatorname{avg}-L R_{T}\left(T_{1}\right) & =\frac{1}{T^{*}} \sum_{T_{2} \in \mathcal{T}_{\epsilon}} F_{T_{2}}\left(T_{1}\right), \\
\exp -L R_{T}\left(T_{1}\right) & =\log \left\{\frac{1}{T^{*}} \sum_{T_{2} \in \mathcal{T}_{\epsilon}} \exp \left(\frac{1}{2} F_{T_{2}}\left(T_{1}\right)\right)\right\},
\end{aligned}
$$

where $\mathcal{T}_{\epsilon}=\left\{T_{2}: \epsilon T \leq T_{2}<T_{1}-\epsilon T, T_{1}+\epsilon T<T_{2} \leq(1-\epsilon) T\right\}, T^{*}$ is the number of $T_{2}$ included in $\mathcal{T}_{\epsilon}$, and $F_{T_{2}}\left(T_{1}\right)$ is the test statistic for the simple null hypothesis of $T_{0}=T_{1}$ against the simple alternative of $T_{0}=T_{2}$, which is given by

$$
F_{T_{2}}\left(T_{1}\right)=\left\{\begin{array}{ll}
\left(\sum_{t=T_{2}+1}^{T_{1}} w_{b, t} \hat{u}_{t}\right)^{\prime}\left(\tilde{\omega}_{u u} \hat{H}\right)^{-1}\left(\sum_{t=T_{2}+1}^{T_{1}} w_{b, t} \hat{u}_{t}\right) & : T_{2}<T_{1} \\
\left(\sum_{t=T_{1}+1}^{T_{2}} w_{b, t} \hat{u}_{t}\right)^{\prime}\left(\tilde{\omega}_{u u} \hat{H}\right)^{-1}\left(\sum_{t=T_{1}+1}^{T_{2}} w_{b, t} \hat{u}_{t}\right) & : T_{1}<T_{T}
\end{array},\right.
$$

\footnotetext{
${ }^{2}$ As $\varepsilon=0.05, \lambda_{1}$ may take values between 0.05 and 0.1 or 0.9 and 0.95 . In these cases, the integral in the limiting expression is taken over the one-sided region $\left(\left\{\lambda_{2}: \lambda_{1}+\varepsilon<\lambda_{2} \leq 1-\varepsilon\right\}\right.$ or $\left.\left\{\lambda_{2}: \varepsilon \leq \lambda_{2} \leq \lambda_{1}-\varepsilon\right\}\right)$ when $\lambda_{1}$ is located near the end points, $\varepsilon \leq \lambda_{1} \leq 2 \varepsilon$ or $1-2 \varepsilon \leq \lambda_{1} \leq 1-\varepsilon$, whereas the range of the integral is two-sided $\left(\left\{\lambda_{2}: \varepsilon \leq \lambda_{2}<\lambda_{1}-\varepsilon, \lambda_{1}+\varepsilon<\lambda_{2} \leq \varepsilon\right\}\right)$ when $\lambda_{1}$ is located far from the end points, $2 \varepsilon<\lambda_{1}<1-2 \varepsilon$. As a result, the response surface regression becomes complicated in this case, as shown by Kurozumi and Yamamoto (2015). To simplify our procedure as far as possible, we restrict the permissible values of the break fraction $\lambda_{1}$ to range from 0.1 to 0.9 .

${ }^{3}$ In the response surface regressions, the maximum relative error, (the fitted value the obtained critical value)/the obtained critical value, among all the regressions was 0.028 .
} 
where $\hat{u}_{t}$ are the regression residuals of $y_{t}$ on $w_{b, t}, w_{b, t}\left(\lambda_{1}\right)$, and $w_{f, t}$,

$$
\hat{H}=\sum_{t=1}^{T} \hat{r}_{t}\left(\lambda_{2}, \lambda\right) \hat{r}_{t}\left(\lambda_{2}, \lambda_{1}\right)^{\prime}
$$

with $\hat{r}_{t}$ being the regression residuals of $r_{t}\left(\lambda_{2}, \lambda_{1}\right)$ on $w_{t}\left(\lambda_{1}\right)$, and $\tilde{\omega}_{u u}$ is a consistent estimator of $\omega_{u u}$. Note that we require $\tilde{\omega}_{u u}$ to be consistent under both the null and the alternative and thus, following Yamamoto (2016), we use the following nonparametric estimator:

$$
\tilde{\omega}_{u u}=\tilde{\gamma}(0)+2 \sum_{j=1}^{T} k(j, m) \tilde{\gamma}(j), \quad \tilde{\gamma}(j)=\frac{1}{T} \sum_{t=j+1}^{T} \tilde{u}_{t} \tilde{u}_{t-j},
$$

where $\tilde{u}_{t}$ are the regression residuals of $y_{t}$ on $w_{b, t}, w_{b, t}\left(\lambda_{1}\right), w_{f, t}$, and $1\left(\left|T_{1}-\hat{T}_{b}\right|>p_{b}\right) w_{b, t}\left(\hat{\lambda}_{b}\right)$ with $\hat{T}_{b}\left(\hat{\lambda}_{b}\right)$ being the break (fraction) estimator by minimizing the sum of the squared residuals, as considered by Kejriwal and Perron (2008a), and $k(j, m)$ is a kernel function such as the Bartlett and quadratic spectral (QS) kernel, as often used in practical analysis, with $m$ being the bandwidth parameter. Note that $w_{b, t}\left(\hat{\lambda}_{b}\right)$ is multiplied by the indicator function $1\left(\left|T_{1}-\hat{T}_{b}\right|>p_{b}\right)$ to avoid the case where $\hat{T}_{b}$ is too close to $T_{1}$ (see Yamamoto (2016) for details).

\section{Finite Sample Property}

In this section, we investigate the finite sample properties of the confidence sets constructed by inverting the three test statistics (19)-(21). We consider the case of only one I(1) regressor, where the data generating process is given by

$$
\begin{aligned}
& \text { Model I-a: } \quad y_{t}=1+z_{t}+1\left(t>\left[\lambda_{0} T\right]\right)\left(\delta_{b, c}+\delta_{b, z} z_{t}\right)+u_{t}, \\
& \text { Model I-b: } \quad y_{t}=1+1\left(t>\left[\lambda_{0} T\right]\right) \delta_{b, c}+z_{t}+u_{t}, \\
& \text { Model II-a: } y_{t}=1+3 \frac{t}{T}+z_{t}+1\left(t>\left[\lambda_{0} T\right]\right)\left(\delta_{b, c}+3 \delta_{b, \tau} \frac{t}{T}+\delta_{b, z} z_{t}\right)+u_{t}, \\
& \text { Model II-b: } \quad y_{t}=1+3 \frac{t}{T}+1\left(t>\left[\lambda_{0} T\right]\right)\left(\delta_{b, c}+3 \delta_{b, \tau} \frac{t}{T}\right)+z_{t}+u_{t} .
\end{aligned}
$$

The I(1) regressor $z_{t}$ and the error term $u_{t}$ are generated by

$$
z_{t}=z_{t-1}+u_{t}^{z}, \quad u_{t}^{z}=\phi_{z} u_{t-1}^{z}+e_{z, t}
$$




$$
\begin{gathered}
u_{t}=\phi_{u} u_{t-1}+e_{u, t} \\
\text { where }\left[\begin{array}{l}
e_{u, t} \\
e_{z, t}
\end{array}\right] \sim \text { i.i.d.N }(0, \Sigma) \quad \text { with } \quad \Sigma=\left[\begin{array}{cc}
1 & \sigma_{z u} \\
\sigma_{z u} & 1
\end{array}\right] .
\end{gathered}
$$

The sample size $T$ is 100 and 300, and we consider the case where a one-time break occurs in the middle of the sample period, $\lambda_{0}=0.5$. We set the magnitude of the break to be $\delta_{b, c}=d / T^{1 / 4}, \delta_{b, \tau}=d / T^{1 / 4}$, and $\delta_{b, z}=d / T^{3 / 4}$ for $d=4,8,12$, and 16, respectively. As a standard case, we consider the case with no serial correlation in $u_{t}^{z}$ and $u_{t}\left(\phi_{z}=\phi_{u}=0\right)$, no endogeneity $\left(\sigma_{z u}=0\right)$, and the leads-and-lags length given by $l=0$ (DGP1); we set $\phi_{z}=0.5$, $\phi_{u}=0$, and $\sigma_{z u}=0$ with $l=0$ to see the effect of the serial correlation in $u_{z, t}$ (DGP2), while DGP3 allows for serial correlation in the regression errors $u_{t}$ by setting $\phi_{z}=0, \phi_{u}=0.5$, $\sigma_{z u}=0$, and $l=0$. The effect of the endogeneity is investigated in DGP4 with $\phi_{z}=\phi_{u}=0$, $\sigma_{z u}=0.5$, where the leads-and-lags order $l$ is selected by the BIC with the maximum lag order set to $\left[8(T / 100)^{1 / 4}\right]$. Finally, DGP5 corresponds to the most general case in which serial correlation and endogeneity are allowed with $\phi_{z}=\phi_{u}=0.5, \sigma_{z u}=0.5$, and $l$ selected by the BIC. ${ }^{4}$ The long-run variance $\omega_{u u}$ is estimated using the QS kernel with the bandwidth selected by the automatic selection rule suggested by Andrews (1991). The confidence level is 0.95 , the number of replications is 5000 , and all the simulations are conducted by using the GAUSS matrix language.

Table 1 shows the coverage rates and lengths of the confidence sets for Model I-a. The column "BLS" is the result based on the confidence interval obtained by using the method proposed by BLS, while the columns "sup," "avg," and "exp" correspond to the confidence sets obtained by inverting the sup-type, average-type, and exponential-type tests, respectively. In DGP1, the coverage rate of BLS for $T=100$ is much smaller than the nominal rate of 0.95 when the magnitude of the break is small, while it becomes close to 0.95 when a large structural change occurs. Similarly, the coverage rates based on the average-type and exponential-type tests tend to be smaller than the nominal rate when $d=4$, while they are not as liberal as BLS. On the contrary, the coverage rate based on the sup-type test is close to 0.95 for all values of $d$. Regarding the length of the confidence set, our three methods tend to construct smaller confidence sets than BLS in GDP1. For example, when $d=16$,

\footnotetext{
${ }^{4}$ We also conducted simulations with a fixed leads-and-lags length using $l=\left[4(T / 100)^{1 / 4}\right]$. The relative performance in this case is similar to the case with $l$ selected by the BIC.
} 
the coverage rates of the four methods are $0.963,0.963,0.938$, and 0.928 , while the lengths of the confidence sets are $0.072,0.061,0.063$, and 0.053 , respectively. However, this is not always the case, as shown in the other data generating processes. For example, in DGP3 with $d=16$, the coverage rate of BLS is smaller than that of the sup-type test, whereas the length of the confidence interval of BLS is shorter than the latter. As a whole, the difference in the sizes of the confidence sets is relatively minor when the empirical coverage rates are close to 0.95 .

From panel DGP2, we can see that the effects of serial correlation in the I(1) regressor on the coverage rates are very minor; they are close to those in DGP1. By contrast, positive serial correlation reduces the lengths of the coverage rates because the variation in the regressors becomes larger than the variance of $u_{t}$ and the relative weight of the noise in the regression becomes smaller, thereby allowing us to estimate the model more efficiently.

On the contrary, serial correlation in the error term does affect the coverage rates, as shown in panel DGP3. For all the methods, the coverage rates become more liberal in this case. However, the coverage rate of the sup-type test recovers to the nominal rate as soon as the magnitude of the break becomes larger. In the case of positive serial correlation in $u_{t}$, the variation of the noise in the regression becomes large and the estimation becomes less efficient. As a result, the lengths of the confidence sets tend to be larger than those in the standard case.

Panel DGP4 suggests that the effect of the endogeneity is relatively minor, at least if we include the leads-and-lags by using the BIC. The coverage rates in DGP4 are close to those in DGP1, while the sizes of the confidence sets are slightly smaller.

DGP5 allows for serial correlation and endogeneity, and the results are a mixture of DGP2 to DGP4. Again, all the methods are liberal when $d=4$; however, the coverage rate of the sup-type test becomes closest to 0.95 when $d \geq 8$, while the difference in the sizes of the confidence sets seems relatively minor.

When $T=300$, all the methods perform better in terms of the coverage rates and the lengths of the confidence sets. In general, the relative performance is preserved in this case.

Table 2 reports the results for Model I-b. In this case, the confidence set of BLS is liberal 
again when the magnitude of the break is small, whereas it becomes too conservative for a large break. For example, the coverage rate of BLS is 0.886 for $d=4$, while it is 1.000 for $d=16$ in DGP1. By contrast, our three methods are better able to control the coverage rate, although it is smaller than the nominal rate when $d=4$ in DGP 3 and DGP5.

Tables 3 and 4 report the results for Models II-a and II-b. In these cases, the coverage rate of BLS becomes close to the nominal rate for the small break, whereas it is conservative for $d \geq 8$ for Model II-a and almost always 1.000 for Model II-b. On the contrary, the coverage rates of our three methods are relatively close to the nominal rate, although the sup-type test tends to be slightly conservative in some cases.

Overall, the sup-type test is the best method for controlling the coverage rate, while the differences in the lengths of the confidence sets are minor, as far as our simulations are concerned.

\section{Empirical Application}

In this section, we demonstrate the performance of our confidence sets by using Russian data. We consider the dependence of the Russian economy on oil prices. This positive longrun dependence originates from neoclassical theory of the capital accumulation channel, as discussed by Esfahani, Mohaddes, and Pesaran (2014), Idrisov, Kazakova and Polbin (2015) and Polbin and Skrobotov (2016). For example, in the Solow growth model, a part of oil revenue is saved in the form of the accumulation of physical capital. The following model is based on Polbin and Skrobotov (2016). This model distinguishes the dynamics of Russian GDP, consumption, and investment from the external economic environment by using a (univariate) cointegrating regression with structural breaks. ${ }^{5}$

First, we consider Russian quarterly real log GDP from 1999 to 2014 in constant 2003 prices (64 observations). Figure 1 illustrates two periods with different real GDP growth rates (refer to the left axis for GDP, consumption, and investment, while the right axis corresponds to oil prices). The first period shows recovering growth from approximately the beginning of the sample to mid-2008 (i.e., when the global financial crisis struck). During

\footnotetext{
${ }^{5}$ Polbin and Skrobotov (2016) also consider the multivariate case based on the error correction model.
} 
this period, the Russian economy grew approximately by $7 \%$ per year. This considerably high growth rate could have been induced by the country's economic recovery after the socalled transformation recession (accompanied by increasing production efficiency) as well as the considerable increase in oil prices (since 2003). After the structural break in 2008, a significant growth rate is observed only briefly after the crisis, 2008-2009 (recovering growth); when GDP reaches the pre-crisis level, the growth rate reduces significantly.

The long-run dependence of real GDP on oil prices can be expressed by the following simple model:

$$
\log y_{t}=\text { deterministic component }+\gamma \log p_{t}^{o i l}+e_{t}
$$

where $y_{t}$ is real output, $p_{t}^{\text {oil }}$ is the real oil price, and $\gamma$ is the long-run price elasticity of real output on the real oil price. If the process $e_{t}$ is stationary, we interpret this relation as cointegration with the long-run dependence of output on oil prices. The structural break in mid-2008 can be induced by the reaction of the oil price dynamics as well as by the change in the long-run growth rate of the structural component of GDP. If only the first source matters, then there is no structural break in the deterministic component. If there is a change in the long-run growth rate of the structural component of GDP, then we can approximate this break as Model II-b:

$$
\log y_{t}=\beta_{b, c}+\beta_{b, \tau} t+1\left(t>\left[\lambda_{0} T\right]\right)\left(\delta_{b, c}+\delta_{b, \tau} t\right)+\gamma \log p_{t}^{o i l}+u_{t}
$$

Throughout this section, we assume that the oil price is a random walk (see, inter alia, Alquist, Kilian, and Vigfusson, 2013). Then, as described by Polbin and Skrobotov (2016), the deterministic component in equation (22) is the unconditional expectation of the GDP growth rate in Russia. Therefore, if there is long-run (cointegrating) dependence of Russian real GDP on oil prices, the long-run GDP growth rate will coincide with the long-run growth rate of the structural component of GDP. A similar type of cointegrating relation for the Russian economy was considered in Rautava (2013) and Kuboniwa (2014). If there is no break, then the economy grows at a rate of $\beta_{b, \tau}$ under fixed oil prices. If there is a break, then the growth rate changes from $\beta_{b, \tau}$ to $\beta_{b, \tau}+\delta_{b, \tau}$. As in Polbin and Skrobotov (2016), according to neoclassical models of exogenous growth, the deterministic trends in (22) can be interpreted as long-run growth in labor efficiency in all sectors of the economy. This long-run 
growth ensures a balanced growth path of real GDP. In the Russian economy, the break in the deterministic component (in particular, the break in trend) in (23) originates from the end of the rapid growth period driven by the substantial reduction in the productivity gap between domestic and foreign economies and need to ensure growth at the expense of their own development.

We can also consider similar regressions for the real consumption and real investment series; real consumption and/or real investment may be cointegrated with oil prices with a structural break in the trend function, as suggested by Figure 1 (see more discussion in Polbin and Skrobotov, 2016).

The data used in this section are logarithms of seasonally adjusted (by X-12-ARIMA) Russian quarterly real GDP, consumption, and investment from 1999 to 2014 in constant 2003 prices (source: Federal State Statistics Service, Russia) and real oil prices (Brent) from 1999 to 2014 (source: International Financial Statistics) deflated by seasonally adjusted CPI in the United States (source: Federal Reserve Economic Data). We investigate the data as follows:

1. Estimate the break date based on a regression without leads-and-lags.

2. Choose the number of leads-and-lags by the BIC (see Choi and Kurozumi, 2012).

3. Re-estimate the break date by using the selected leads-and-lags.

4. Test for the null hypothesis of cointegration with a break by using the test proposed by Arai and Kurozumi (2007).

5. If we do not reject the null hypothesis of cointegration, then construct the confidence set based on the leads-and-lags regression and estimated break date.

The first two steps imply that we choose the leads-and-lags under the alternative. By using the selected leads-and-lags, we re-estimate the break date in step 3. This estimated break date is then used for the LM-type test for the null hypothesis of cointegration with a break proposed by Arai and Kurozumi (2007) in step 4. We construct the confidence set in the final step if the null hypothesis of cointegration is not rejected. 
Table 5 reports the estimated break dates. For GDP and consumption, they are in 2008, whereas a structural change may have occurred in 2006 in the relationship between investment and oil prices. By using the estimated break dates, we test for the null hypothesis of cointegration with a break for each series where the long-run variance is estimated based on the kernel method by using the QS kernel and data-dependent bandwidth rule proposed by Andrews (1991), and we cannot reject the null hypothesis. ${ }^{6}$ For completeness, we also test for the null hypothesis of cointegration without a break as proposed by Shin (1994), and we reject the null for GDP and investment at the $5 \%$ significance level and for consumption at the $10 \%$ significance level. ${ }^{7}$ These results suggest a cointegrating relationship between Russian macroeconomic variables and oil prices because it is known that if series are cointegrated with a break, we do reject the null hypothesis of cointegration if we use the usual test without a break (Shin's test), whereas we fail to reject the null hypothesis if we appropriately introduce the break into the model, as in the case of unit root tests (Perron, 1989). Furthermore, we test for parameter stability by using the LM and Wald tests by plugging in the estimated break date as if it were the true one, which is theoretically supported by BLS. We find that both tests reject the null hypothesis at the $1 \%$ significance level for all the series. Note that while the LM test for parameter stability is known to suffer from the non-monotonic power problem, as shown by Vogelsang (1999), it still strongly rejects the parameter stability hypothesis. From these test results, we proceed with a cointegration model with a break as given by (23).

Once evidence of cointegration with a break is observed, we next construct confidence sets, as summarized in Figure 2. We select the different lengths of leads-and-lags by using the BIC, which are (leads, lags $)=(0,0)$ for the regression with GDP, $(1,5)$ for that with consumption, and $(0,0)$ for that with investment. In the figure, the bold vertical line shows the estimated break date, which is also reported in Table 5. For the cointegrating regression with GDP reported in Figure 2(i), the sup-type and average-type tests return the same confidence sets ranging from 2008Q1 to 2009Q1, while those based on the exponential-type test and BLS are

\footnotetext{
${ }^{6}$ Model (23) is not included in Arai and Kurozumi (2007) and we obtained asymptotic critical values for the given break fractions from the simulations.

${ }^{7}$ We also employed the long-run variance estimators used in Carrion-i-Silvestre and Sansó (2006) and obtained the same results.
} 
from 2008Q2 to 2008Q4. As observed in the simulation section, the coverage rate based on the sup-type test tends to be close to the nominal confidence level while it may be difficult to control the coverage rate for the other three methods; in particular, the coverage rate of BLS becomes either liberal or conservative depending on the case. Taking this result into account, the break point could be located outside the confidence interval estimated by BLS. Similarly, the confidence sets for the break dates in the cointegrating regressions with consumption and investment are reported in Figures 2(ii) and 2(iii). Similar to the regression with GDP, the confidence set based on the sup-type test is wider than the others; hence, considering the stable coverage rate of the sup-type test, the break point could again be located outside the periods covered by the other methods. We also observe that the confidence set based on our methods could return an asymmetric interval, while that based on BLS is always symmetric. To summarize, Russian quarterly macroeconomic variables are cointegrated with oil prices with a break and the possible break date could range more than one year.

\section{Concluding Remarks}

In this paper, we proposed constructing a confidence set for the break date in cointegrating regressions by inverting the test that maximizes the weighted average of power. We derived the limiting distribution of the test for the break location, which depends on whether a linear trend is included and/or the coefficients associated with the $\mathrm{I}(1)$ regressors sustain a structural change. By Monte Carlo simulations, we showed that the confidence set based on our test, particularly the sup-type test, can better control the coverage rate, while the size of the confidence set is at least as small as the existing method. We then applied our method to analyze the Russian economy and showed that it is informative for the location of the break date. 


\section{References}

[1] Alquist, R., L. Kilian and R. J. Vigfusson (2013). Forecasting the Price of Oil. Handbook of Economic Forecasting 2, 427-507.

[2] Andrews, D. W. K. (1991). Heteroskedasticity and Autocorrelation Consistent Covariance Matrix Estimation. Econometrica 59, 817-858.

[3] Andrews, D. W. K. (1993). Tests for Parameter Instability and Structural Change with Unknown Change Point. Econometrica 61, 821-856 (Erratum, 71, 395-397).

[4] Andrews, D. W. K. and W. Ploberger (1994). Optimal Tests When a Nuisance Parameter Is Present Only Under the Alternative, Econometrica 62, 1383-1414.

[5] Arai, Y. and E. Kurozumi (2007). Testing for the Null Hypothesis of Cointegration with a Structural Break. Econometric Reviews 26, 705-7390.

[6] Bai, J. (1997). Estimation of a Change Point in Multiple Regression Models. Review of Economics and Statistics 79, 551-563.

[7] Bai, J., R. L. Lumsdaine and J. H. Stock (1998). Testing For and Dating Common Breaks in Multivariate Time Series. Review of Economic Studies 65, 395-432.

[8] Bai, J. and P. Perron (1998). Estimating and Testing Linear Models with Multiple Structural Changes. Econometrica 66, 47-78.

[9] Campos, J., N. R. Ericsson and D. F. Hendry (1996). Cointegration Tests in the Presence of Structural Breaks. Journal of Econometrics 70, 187-220.

[10] Carrion-i-Silvestre J. and A. J. Sansó (2006). Testing the Null of Cointegration with Structural Breaks. Oxford Bulletin of Economics and Statistics 68, 623-646.

[11] Chang, S. Y. and P. Perron (2015). A Comparison of Alternative Methods to Construct Confidence Intervals for the Estimate of a Break Date in Linear Regression Models. Econometric Reviews, DOI: 10.1080/07474938.2015.1122142. 
[12] Choi, I. and E. Kurozumi (2012). Model Selection Criteria for the Leads-and-lags Cointegrating Regression. Journal of Econometrics 169, 224-238.

[13] Elliott, G. and U. K. Müller (2007). Confidence Sets for the Date of a Single Break in Linear Time Series Regressions. Journal of Econometrics 141, 1196-1218.

[14] Eo, Y. and J. Morley (2015). Likelihood-ratio-based Confidence Sets for the Timing of Structural Breaks. Quantitative Economics 6, 463-497.

[15] Esfahani H. S., K. Mohaddes and M. H. Pesaran (2014). An Empirical Growth Model for Major Oil Exporters, Journal of Applied Econometrics 29, 1-21.

[16] Gregory, A. W. and B. E. Hansen (1996a). Residual-based Tests for Cointegration in Models with Regime Shifts. Journal of Econometrics 70, 99-126.

[17] Gregory, A. W. and B. E. Hansen (1996b). Tests for Cointegration in Models with Regime and Trend Shifts. Oxford Bulletin of Economics and Statistics 58, 555-560.

[18] Harvey, D. I. and S. J. Leybourne (2015). Confidence Sets for the Date of a Break in Level and Trend When the order of integration is unknown. Journal of Econometrics 184, 262-279.

[19] Hayakawa, K. and E. Kurozumi (2008). The Role of Leads in the Dynamic OLS Estimation of Cointegrating Regression Models. Mathematics and Computers in Simulation $79,555-560$.

[20] Idrisov, G., M. Kazakova, and A. Polbin (2015). A theoretical interpretation of the oil prices impact on economic growth in contemporary Russia. Russian Journal of Economics 1, 257-272.

[21] Kejriwal, M. and P. Perron (2008a). The Limiting Distribution of the Estimates in Cointegrated Regression Models with Multiple Structural Changes. Journal of Econometrics $146,59-73$. 
[22] Kejriwal, M. and P. Perron (2008b). Data Dependent Rules for Selection of the Number of Leads and Lags in the Dynamic OLS Cointegrating Regression. Econometric Theory $24,1425-1441$.

[23] Kejriwal, M. and P. Perron (2010). Testing for Multiple Structural Changes in Cointegrated Regression Models. Journal of Business 83 Economic Statistics 28, 503-522.

[24] Kuboniwa, M. (2014). A Comparative Analysis of the Impact of Oil Prices on Oil-Rich Emerging Economies in the Pacific Rim. Journal of Comparative Economics 42, 328-339.

[25] Kurozumi, E. (2016). Construction of Confidence Sets for the Break Date in Regression Models with Non-homogeneous Regressors (in Japanese). Journal of the Japan Statistical Society, forthcoming.

[26] Kurozumi, E. and Y. Yamamoto (2015). Confidence Sets for the Break Date Based on Optimal Tests. Econometrics Journal 18, 412-435.

[27] Perron, P. (1989). The Great Crash, the Oil Price shock, and the Unit Root Hypothesis. Econometrica 57, 1361-1401 (Erratum, 61, 249-249).

[28] Phillips, P. C. B. and M. Loretan (1991). Estimating Long-run Economic Equilibria. Review of Economic Studies 58, 407-436.

[29] Polbin A. and A. Skrobotov (2016). Testing for Structural Breaks in the Long-run Growth Rate of the Russian Economy (in Russian). Mimeo.

[30] Rautava, J. (2013). Oil Prices, Excess Uncertainty and Trend Growth. Focus on European Economic Integration 4, 77-87.

[31] Saikkonen, P. (1991). Asymptotically Efficient Estimation of Cointegration Regressions. Econometric Theory 7, 1-21.

[32] Shin, Y. (1994). A Residual- Based Test of the Null of Cointegration against the Alternative of No Cointegration. Econometric Theory 10, 91-115.

[33] Stock, J. H. and M. W. Watson (1993). A Simple Estimator of Cointegrating Vectors in Higher Order Integrated Systems. Econometrica 61, 783-820. 
[34] Vogelsang, T. J. (1999). Sources of Nonmonotonic Power When Testing for a Shift in Mean of a Dynamic Time Series. Journal of Econometrics 88, 283-299.

[35] Yamamoto, Y. (2016). A Modified Confidence Set for the Structural Break Date in Linear Regression Models. Econometric Reviews, DOI: 10.1080/00927872.2016.1178892. 


\section{Appendix A}

Here, we prove the theorem for Model II-c. The other cases can be proven in the same manner. For the test statistic $\widetilde{L R}_{T}\left(\lambda_{1}\right)$, we note that

$$
\begin{aligned}
& y^{\prime} M_{w_{1}} R\left(\lambda_{2}, \lambda_{1}\right)\left(R\left(\lambda_{2}, \lambda_{1}\right)^{\prime} M_{w_{1}} R\left(\lambda_{2}, \lambda_{1}\right)\right)^{-1} R\left(\lambda_{2}, \lambda_{1}\right)^{\prime} M_{w_{1}} y \\
& \quad=\left(D_{T}^{\prime} R\left(\lambda_{2}, \lambda_{1}\right)^{\prime} M_{\tilde{w}_{1}} y\right)^{\prime}\left(D_{T}^{\prime} R\left(\lambda_{2}, \lambda_{1}\right)^{\prime} M_{\tilde{w}_{1}} R\left(\lambda_{2}, \lambda_{1}\right) D_{T}\right)^{-1}\left(D_{T}^{\prime} R\left(\lambda_{2}, \lambda_{1}\right)^{\prime} M_{\tilde{w}_{1}} y\right),
\end{aligned}
$$

where $D_{T}=\operatorname{diag}\left\{T^{-1 / 2}, T^{-3 / 2}, T^{-1} \Omega_{b b}^{-1 / 2}\right\}$ and $M_{\tilde{w}_{1}}=\tilde{W}\left(\lambda_{1}\right)\left(\tilde{W}\left(\lambda_{1}\right)^{\prime} \tilde{W}\left(\lambda_{1}\right)\right)^{-1} \tilde{W}\left(\lambda_{1}\right)^{\prime}$ with $\tilde{W}\left(\lambda_{1}\right)=\left[W_{b}-W_{b}\left(\lambda_{1}\right), W_{b}\left(\lambda_{1}\right), W_{f}\right]$. Note that $D_{T}$ adjusts the convergence order as well as transforms the $\mathrm{I}(1)$ regressors $z_{b, t}$ in $R\left(\lambda_{2}, \lambda_{1}\right)$ to $\tilde{z}_{b, t}=\Omega_{b b}^{-1 / 2} z_{b, t}$ and

$$
\frac{1}{\sqrt{T}} \tilde{z}_{b,[r T]}=\frac{1}{\sqrt{T}} \Omega_{b b}^{-1 / 2} z_{b, t} \Rightarrow B_{b}(r),
$$

meaning that the resulting distribution becomes free of the nuisance parameters.

Let us consider the case where $\lambda_{2}<\lambda_{1}$. We first note that

$$
\begin{aligned}
& D_{T}^{\prime} R\left(\lambda_{2}, \lambda_{1}\right)^{\prime} M_{\tilde{w}_{1}} R\left(\lambda_{2}, \lambda_{1}\right) D_{T} \\
& =D_{T}^{\prime} R\left(\lambda_{2}, \lambda_{1}\right)^{\prime} R\left(\lambda_{2}, \lambda_{1}\right) D_{T} \\
& -D_{T}^{\prime} R\left(\lambda_{2}, \lambda_{1}\right)^{\prime} \tilde{W}\left(\lambda_{1}\right) \tilde{D}_{T}\left(\tilde{D}_{T}^{\prime} \tilde{W}\left(\lambda_{1}\right)^{\prime} \tilde{W}\left(\lambda_{1}\right) \tilde{D}_{T}\right)^{-1} \tilde{D}_{T}^{\prime} \tilde{W}\left(\lambda_{1}\right)^{\prime} R\left(\lambda_{2}, \lambda_{1}\right) D_{T},
\end{aligned}
$$

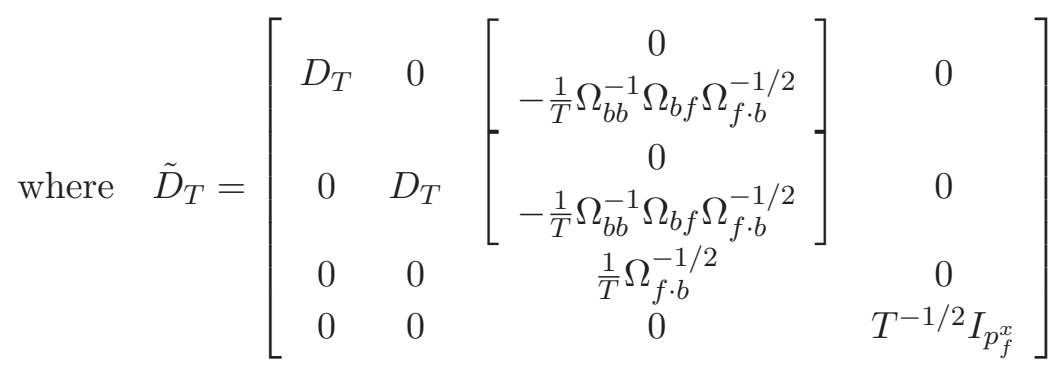

with $\Omega_{f \cdot b}=\Omega_{f f}-\Omega_{f b} \Omega_{b b}^{-1} \Omega_{b f}$ as defined before Theorem 1. Note that the matrix $\tilde{D}_{T}$ transforms $z_{f,[r T]}$ in $\tilde{W}\left(\lambda_{1}\right)$ to $\tilde{z}_{f \cdot b, t}=\Omega_{f \cdot b}^{-1 / 2}\left(z_{f, t}-\Omega_{f b} \Omega_{b b}^{-1} z_{b, t}\right)$ and

$$
\frac{1}{\sqrt{T}} \tilde{z}_{f \cdot b,[r T]}=\frac{1}{\sqrt{T}} \Omega_{f \cdot b}^{-1 / 2} z_{f,[r T]}-\frac{1}{T} \Omega_{f \cdot b}^{-1 / 2} \Omega_{f b} \Omega_{b b}^{-1} z_{b,[r T]} \Rightarrow B_{f \cdot b}(r),
$$

which is independent of $B_{b}(r)$ and $B_{u}(r)$. 
Then, under Assumption 1, we can see that

$$
\begin{aligned}
& D_{T}^{\prime} R\left(\lambda_{2}, \lambda_{1}\right)^{\prime} R\left(\lambda_{2}, \lambda_{1}\right) D_{T} \\
& =D_{T}^{\prime} \sum_{t=T_{2}+1}^{T_{1}} w_{b, t} w_{b, t}^{\prime} D_{T} \\
& =\left[\begin{array}{ccc}
\frac{T_{1}-T_{2}}{T} & \frac{1}{T^{2}} \sum_{t=T_{2}+1}^{T_{1}} t & \frac{1}{T \sqrt{T}} \sum_{t=T_{2}+1}^{T_{1}} \tilde{z}_{b, t}^{\prime} \\
\frac{1}{T^{2}} \sum_{t=T_{2}+1}^{T_{1}} t & \frac{1}{T^{3}} \sum_{t=T_{2}+1}^{T_{1}} t^{2} & \frac{1}{T^{2} \sqrt{T}} \sum_{t=T_{2}+1}^{T_{1}} t \tilde{z}_{b, t}^{\prime} \\
\frac{1}{T \sqrt{T}} \sum_{t=T_{2}+1}^{T_{1}} \tilde{z}_{b, t} & \frac{1}{T^{2} \sqrt{T}} \sum_{t=T_{2}+1}^{T_{1}} t \tilde{z}_{b, t} & \frac{1}{T^{2}} \sum_{t=T_{2}+1}^{T_{1}} \tilde{z}_{b, t} \tilde{z}_{b, t}^{\prime}
\end{array}\right] \\
& \Rightarrow\left[\begin{array}{ccc}
\lambda_{1}-\lambda_{2} & \frac{\lambda_{1}^{2}-\lambda_{2}^{2}}{2} & \int_{\lambda_{2}}^{\lambda_{1}} B_{b}(r)^{\prime} d r \\
\frac{\lambda_{1}^{2}-\lambda_{2}^{2}}{2} & \frac{\lambda_{1}^{3}-\lambda_{2}^{3}}{3} & \int_{\lambda_{2}}^{\lambda_{1}} r B_{b}(r)^{\prime} d r \\
\int_{\lambda_{2}}^{\lambda_{1}} B_{b}(r) d r & \int_{\lambda_{2}}^{\lambda_{1}} r B_{b}(r) d r & \int_{\lambda_{2}}^{\lambda_{1}} B_{b}(r) B_{b}(r)^{\prime} d r
\end{array}\right] \\
& =\int_{\lambda_{2}}^{\lambda_{1}} \tilde{B}_{b}(r) \tilde{B}_{b}(r)^{\prime} d r,
\end{aligned}
$$

where $\tilde{B}_{b}(r)=\left[1, r, B_{b}(r)^{\prime}\right]^{\prime}$. In the same manner, we have

$$
\begin{aligned}
& D_{T}^{\prime} R\left(\lambda_{2}, \lambda_{1}\right)^{\prime} \tilde{W}\left(\lambda_{1}\right) \tilde{D}_{T} \\
& =D_{T}^{\prime} \sum_{t=T_{2}+1}^{T_{1}} w_{b, t} \tilde{w}_{t}\left(\lambda_{1}\right)^{\prime} \tilde{D}_{T} \\
& =\left[\begin{array}{ccc}
\frac{T_{1}-T_{2}}{T} & \frac{1}{T^{2}} \sum_{t=T_{2}+1}^{T_{1}} t & \frac{1}{T \sqrt{T}} \sum_{t=T_{2}+1}^{T_{1}} \tilde{z}_{b, t}^{\prime} \\
\frac{1}{T^{2}} \sum_{t=T_{2}+1}^{T_{1}} t & \frac{1}{T^{3}} \sum_{t=T_{2}+1}^{T_{1}} t^{2} & \frac{1}{T^{2} \sqrt{T}} \sum_{t=T_{2}+1}^{T_{1}} t \tilde{z}_{b, t}^{\prime} \\
\frac{1}{T \sqrt{T}} \sum_{t=T_{2}+1}^{T_{1}} \tilde{z}_{b, t} & \frac{1}{T^{2}} \sum_{t=T_{2}+1}^{T_{1}} t \tilde{z}_{b, t} & \frac{1}{T^{2}} \sum_{t=T_{2}+1}^{T_{1}} \tilde{z}_{b, t} \tilde{z}_{b, t}^{\prime}
\end{array}\right.
\end{aligned}
$$

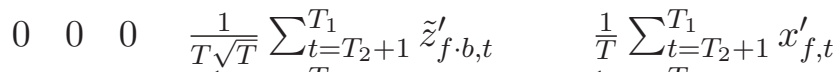

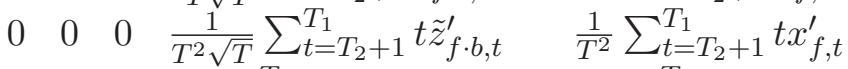

$$
\begin{aligned}
& \left.\begin{array}{lllll}
0 & 0 & 0 & \frac{1}{T^{2}} \sum_{t=T_{2}+1}^{T_{1}} \tilde{z}_{b, f} \tilde{z}_{f \cdot b, t}^{\prime} & \frac{1}{T \sqrt{T}} \sum_{t=T_{2}+1}^{T_{1}} \tilde{z}_{b, t} x_{f, t}^{\prime}
\end{array}\right] \\
& \Rightarrow\left[\int_{\lambda_{2}}^{\lambda_{1}} \tilde{B}_{b}(r) \tilde{B}_{b}(r)^{\prime} d r, 0, \int_{\lambda_{2}}^{\lambda_{1}} \tilde{B}_{b}(r) B_{f \cdot b}(r)^{\prime} d r, 0\right]=\left[J_{1}\left(\lambda_{1}, \lambda_{2}\right), 0\right],
\end{aligned}
$$


and

$$
\begin{aligned}
\tilde{D}_{T}^{\prime} \tilde{W}\left(\lambda_{1}\right)^{\prime} \tilde{W}\left(\lambda_{1}\right) \tilde{D}_{T} \\
=\tilde{D}_{T}^{\prime} \sum_{t=1}^{T} \tilde{w}_{t}\left(\lambda_{1}\right) \tilde{w}_{t}\left(\lambda_{1}\right)^{\prime} \tilde{D}_{T} \\
\Rightarrow\left[\begin{array}{cccc}
\int_{0}^{\lambda_{1}} \tilde{B}_{b}(r) \tilde{B}_{b}(r)^{\prime} d r & 0 & \int_{0}^{\lambda_{1}} \tilde{B}_{b}(r) B_{f \cdot b}(r)^{\prime} d r & 0 \\
0 & \int_{\lambda_{1}}^{1} \tilde{B}_{b}(r) \tilde{B}_{b}(r)^{\prime} d r & \int_{\lambda_{1}}^{1} \tilde{B}_{b}(r) B_{f \cdot b}(r)^{\prime} d r & 0 \\
\int_{0}^{\lambda_{1}} B_{f \cdot b}(r) \tilde{B}_{b}(r)^{\prime} d r & \int_{\lambda_{1}}^{1} B_{f \cdot b}(r) \tilde{B}_{b}(r)^{\prime} d r & \int_{0}^{1} B_{f \cdot b}(r) B_{f \cdot b}(r)^{\prime} d r & 0 \\
0 & 0 & 0 & \Sigma_{x}
\end{array}\right] \\
=\left[\begin{array}{cc}
K\left(\lambda_{1}\right) & 0 \\
0 & \Sigma_{x}
\end{array}\right] .
\end{aligned}
$$

Then, from (24)-(26), we have

$$
D_{T}^{\prime} R\left(\lambda_{2}, \lambda_{1}\right)^{\prime} M_{\tilde{w}_{1}} R\left(\lambda_{2}, \lambda_{1}\right) D_{T} \Rightarrow \int_{\lambda_{2}}^{\lambda_{1}} \tilde{B}_{b}(r) \tilde{B}_{b}(r)^{\prime} d r-J_{1}\left(\lambda_{1}, \lambda_{2}\right)^{\prime} K\left(\lambda_{1}\right)^{-1} J_{1}\left(\lambda_{1}, \lambda_{2}\right) .
$$

Next, since $y=W\left(\lambda_{1}\right) \beta+u$ under the null hypothesis of $\lambda_{0}=\lambda_{1}$, as can be seen in (13), we have

$$
\begin{aligned}
& D_{T}^{\prime} R\left(\lambda_{2}, \lambda_{1}\right)^{\prime} M_{\tilde{w}_{1}} y \\
& \quad=D_{T}^{\prime} R\left(\lambda_{2}, \lambda_{1}\right)^{\prime} u-D_{T}^{\prime} R\left(\lambda_{2}, \lambda_{1}\right)^{\prime} \tilde{W}\left(\lambda_{1}\right) \tilde{D}_{T}\left(\tilde{D}_{T}^{\prime} \tilde{W}\left(\lambda_{1}\right)^{\prime} \tilde{W}\left(\lambda_{1}\right) \tilde{D}_{T}\right)^{-1} \tilde{D}_{T}^{\prime} \tilde{W}\left(\lambda_{1}\right)^{\prime} u .
\end{aligned}
$$

Again, under Assumption 1, we have

$$
\begin{aligned}
D_{T}^{\prime} R\left(\lambda_{2}, \lambda_{1}\right)^{\prime} u & =D_{T}^{\prime} \sum_{t=T_{2}+1}^{T_{1}} w_{b, t} u_{t} \\
& =\left[\begin{array}{c}
\frac{1}{\sqrt{T}} \sum_{t=T_{2}+1}^{T_{1}} u_{t} \\
\frac{1}{T \sqrt{T}} \sum_{t=T_{2}+1}^{T_{1}} t u_{t} \\
\frac{1}{T} \sum_{t=T_{2}+1}^{T_{1}} \tilde{z}_{b, t} u_{t}
\end{array}\right] \\
& \Rightarrow \omega_{u u}^{1 / 2} \int_{\lambda_{2}}^{\lambda_{1}} \tilde{B}_{b}(r) d B_{u}(r),
\end{aligned}
$$

and

$$
\begin{aligned}
\tilde{D}_{T}^{\prime} \tilde{W}\left(\lambda_{1}\right)^{\prime} u= & \tilde{D}_{T}^{\prime} \sum_{t=1}^{T} \tilde{w}_{t}\left(\lambda_{1}\right) u_{t} \\
\Rightarrow & {\left[\begin{array}{c}
\omega_{u u}^{1 / 2} \int_{0}^{\lambda_{1}} \tilde{B}_{b}(r) d B_{u}(r) \\
\omega_{u u}^{1 / 2} \int_{\lambda_{1}}^{1} \tilde{B}_{b}(r) d B_{u}(r) \\
\omega_{u u}^{1 / 2} \int_{0}^{1} B_{f \cdot b}(r) d B_{u}(r) \\
O_{p}(1)
\end{array}\right]=\left[\begin{array}{c}
\omega_{u u}^{1 / 2} L\left(\lambda_{1}\right) \\
O_{p}(1)
\end{array}\right] . }
\end{aligned}
$$


From (25), (26), (28), and (29), we obtain

$$
D_{T}^{\prime} R\left(\lambda_{2}, \lambda_{1}\right)^{\prime} M_{\tilde{w}_{1}} y \Rightarrow \omega_{u u}^{1 / 2}\left(\int_{\lambda_{2}}^{\lambda_{1}} \tilde{B}_{b}(r) d B_{u}(r)-J_{1}\left(\lambda_{1}, \lambda_{2}\right) K\left(\lambda_{1}\right)^{-1} L\left(\lambda_{1}\right)\right) .
$$

The limiting distribution of the test statistic is obtained from (27) and (30) for $\lambda_{2}<\lambda_{1}$. The result for $\lambda_{2}>\lambda_{1}$ is obtained similarly.

The theorem for the other models is proved in the same manner. 


\section{Appendix B}

Table B.1: Coefficients of the response surface regressions (Model I-a)

\begin{tabular}{|c|c|c|c|c|c|c|c|c|}
\hline & \multicolumn{2}{|c|}{ confidence level $=0.9$} & \multicolumn{6}{|c|}{ confidence level $=0.95$} \\
\hline & $p_{b}^{z}=1$ & 2 & 3 & 4 & $p_{b}^{z}=1$ & 2 & 3 & 4 \\
\hline \multicolumn{9}{|c|}{ sup- $L R_{T}$} \\
\hline$a_{0}$ & 695.025 & 230.321 & 611.599 & 446.265 & 527.107 & 194.143 & 614.973 & 295.263 \\
\hline$a_{-1}$ & -682.721 & -215.312 & -594.541 & -427.085 & -513.026 & -177.273 & -596.134 & -274.177 \\
\hline$a_{1}$ & -677.973 & -216.055 & -591.452 & -425.746 & -511.464 & -180.072 & -593.118 & -274.051 \\
\hline$a_{2}$ & 614.214 & 205.077 & 543.605 & 394.135 & 469.267 & 177.585 & 546.530 & 255.638 \\
\hline$a_{3}$ & -359.150 & -140.560 & -332.187 & -247.654 & -280.410 & -128.900 & -335.239 & -166.605 \\
\hline \multicolumn{9}{|c|}{$\operatorname{avg}-L R_{T}$} \\
\hline$a_{0}$ & -67.517 & -4.742 & 38.664 & -44.795 & -25.396 & 14.564 & -1.411 & 13.688 \\
\hline$a_{-1}$ & 70.655 & 9.170 & -33.019 & 51.692 & 29.043 & -9.570 & 7.700 & -6.094 \\
\hline$a_{1}$ & 69.984 & 9.347 & -32.545 & 50.884 & 28.021 & -8.879 & 8.139 & -6.433 \\
\hline$a_{2}$ & -60.633 & -7.170 & 32.583 & -39.274 & -16.550 & 10.989 & -2.378 & 15.951 \\
\hline$a_{3}$ & 35.541 & 7.592 & -16.432 & 20.054 & 5.967 & -2.758 & 3.964 & -11.637 \\
\hline \multicolumn{9}{|c|}{$\exp -L R_{T}$} \\
\hline$a_{0}$ & 2.798 & -12.572 & 53.978 & 36.773 & 97.372 & 102.603 & 1.783 & 129.459 \\
\hline$a_{-1}$ & -0.123 & 16.283 & -49.374 & -31.246 & -94.085 & -98.245 & 3.528 & -123.203 \\
\hline$a_{1}$ & -0.384 & 15.638 & -49.078 & -31.513 & -93.667 & -97.790 & 3.760 & -121.969 \\
\hline$a_{2}$ & 1.194 & -13.246 & 44.108 & 30.038 & 84.168 & 88.665 & -3.895 & 109.088 \\
\hline$a_{3}$ & -2.076 & 5.542 & -26.456 & -21.076 & -45.404 & -50.997 & 0.252 & -62.366 \\
\hline
\end{tabular}


Table B.2: Coefficients of the response surface regressions (Model I-b)

\begin{tabular}{|c|c|c|c|c|c|c|c|c|}
\hline & \multicolumn{2}{|c|}{ confidence level $=0.9$} & \multicolumn{6}{|c|}{ confidence level $=0.95$} \\
\hline & $p_{f}^{z}=1$ & 2 & 3 & 4 & $p_{f}^{z}=1$ & 2 & 3 & 4 \\
\hline \multicolumn{9}{|c|}{ sup- $L R_{T}$} \\
\hline$a_{0}$ & 266.232 & 63.571 & 155.596 & 182.708 & 548.169 & 347.244 & 190.643 & 10.798 \\
\hline$a_{-1}$ & -256.956 & -54.017 & -145.772 & -172.655 & -537.386 & -336.214 & -179.279 & 0.931 \\
\hline$a_{1}$ & -256.999 & -54.097 & -144.358 & -170.615 & -534.243 & -333.991 & -177.364 & 0.950 \\
\hline$a_{2}$ & 242.167 & 53.478 & 131.757 & 154.259 & 486.989 & 306.444 & 163.143 & 1.629 \\
\hline$a_{3}$ & -153.729 & -41.001 & -79.626 & -91.230 & -283.650 & -182.508 & -99.920 & -7.094 \\
\hline \multicolumn{9}{|c|}{ avg- $L R_{T}$} \\
\hline$a_{0}$ & -28.100 & -87.289 & -44.889 & -31.668 & 77.461 & -53.186 & -71.531 & -89.269 \\
\hline$a_{-1}$ & 29.902 & 89.059 & 46.595 & 33.348 & -75.256 & 55.330 & 73.620 & 91.285 \\
\hline$a_{1}$ & 29.896 & 88.601 & 46.847 & 33.434 & -75.056 & 55.193 & 73.281 & 91.366 \\
\hline$a_{2}$ & -29.054 & -81.589 & -45.638 & -32.429 & 69.716 & -51.809 & -67.679 & -87.243 \\
\hline$a_{3}$ & 21.657 & 48.817 & 30.398 & 21.538 & -37.131 & 34.100 & 41.466 & 55.902 \\
\hline \multicolumn{9}{|c|}{$\exp -L R_{T}$} \\
\hline$a_{0}$ & 14.082 & 5.506 & -32.751 & -46.655 & 146.433 & 33.369 & -17.908 & -102.067 \\
\hline$a_{-1}$ & -12.478 & -3.910 & 34.351 & 48.281 & -144.355 & -31.269 & 20.016 & 104.195 \\
\hline$a_{1}$ & -12.745 & -3.912 & 34.383 & 48.052 & -143.521 & -31.047 & 19.990 & 103.545 \\
\hline$a_{2}$ & 12.548 & 3.865 & -31.986 & -43.875 & 129.638 & 27.262 & -17.976 & -94.010 \\
\hline$a_{3}$ & -7.824 & -2.851 & 18.592 & 24.749 & -71.139 & -13.809 & 9.694 & 53.160 \\
\hline
\end{tabular}


Table B.3: Coefficients of the response surface regressions (Model I-c)

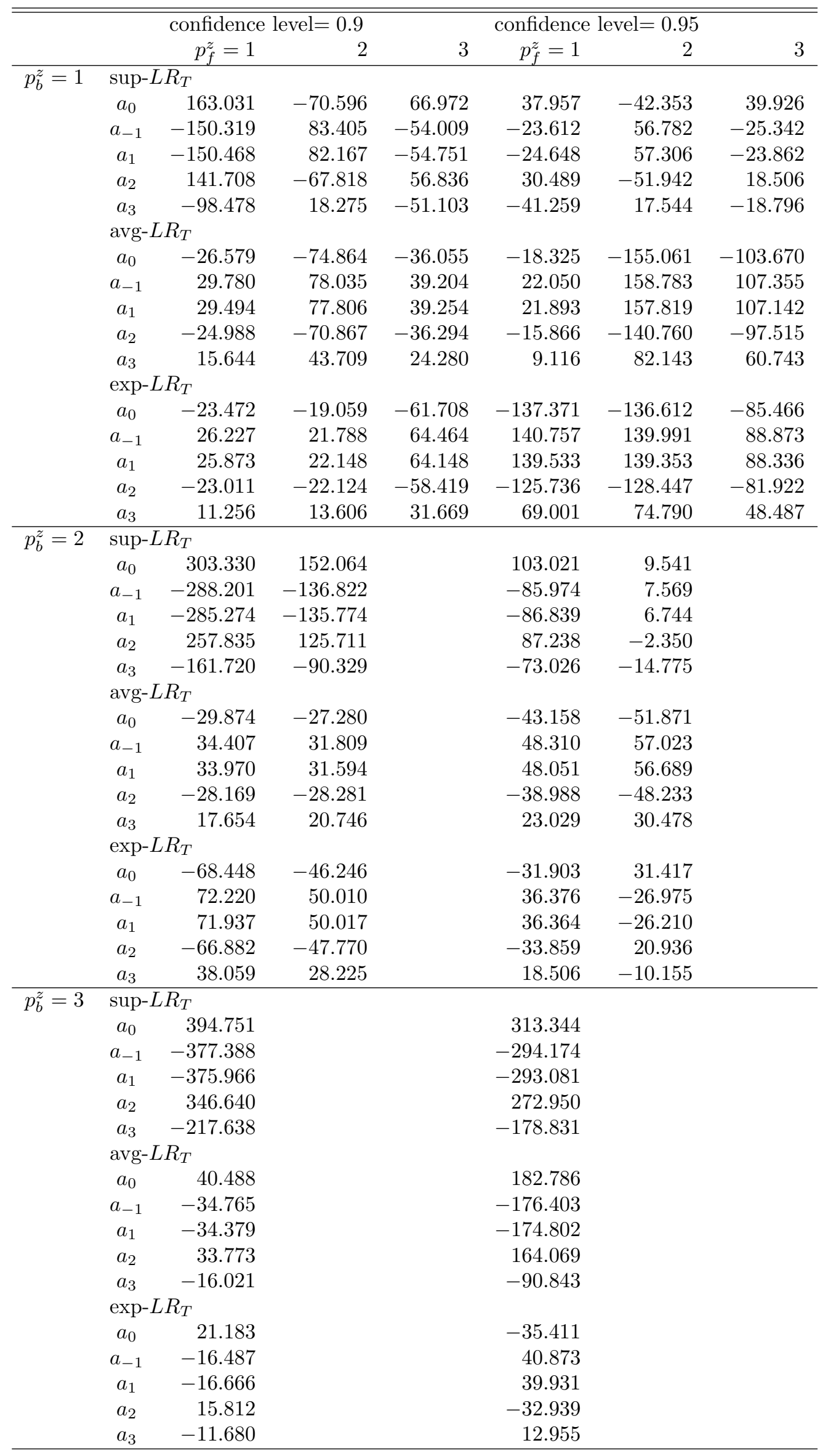


Table B.4: Coefficients of the response surface regressions (Model II-a)

\begin{tabular}{|c|c|c|c|c|c|c|c|c|}
\hline & \multicolumn{2}{|c|}{ confidence level $=0.9$} & \multicolumn{6}{|c|}{ confidence level $=0.95$} \\
\hline & $p_{b}^{z}=1$ & 2 & 3 & 4 & $p_{b}^{z}=1$ & 2 & 3 & 4 \\
\hline \multicolumn{9}{|c|}{ sup- $L R_{T}$} \\
\hline$a_{0}$ & 492.130 & 369.181 & 515.946 & 423.522 & 470.123 & 304.997 & 259.217 & 328.062 \\
\hline$a_{-1}$ & -476.967 & -351.765 & -496.675 & -402.336 & -453.083 & -285.782 & -238.126 & -304.848 \\
\hline$a_{1}$ & -474.993 & -350.083 & -493.984 & -400.520 & -451.868 & -284.049 & -236.043 & -303.513 \\
\hline$a_{2}$ & 439.066 & 317.486 & 451.864 & 368.371 & 414.827 & 258.147 & 214.645 & 279.579 \\
\hline$a_{3}$ & -273.010 & -192.033 & -275.223 & -230.669 & -252.008 & -161.675 & -138.459 & -178.937 \\
\hline \multicolumn{9}{|c|}{$\operatorname{avg}-L R_{T}$} \\
\hline$a_{0}$ & -46.922 & -1.169 & 36.556 & 90.531 & 4.796 & -13.991 & 105.999 & 108.377 \\
\hline$a_{-1}$ & 51.387 & 6.836 & -29.658 & -82.435 & 0.249 & 20.297 & -98.391 & -99.491 \\
\hline$a_{1}$ & 50.572 & 7.308 & -29.493 & -82.117 & 0.210 & 21.355 & -97.361 & -99.343 \\
\hline$a_{2}$ & -40.291 & -4.427 & 31.347 & 80.290 & 6.116 & -17.640 & 93.101 & 99.859 \\
\hline$a_{3}$ & 21.002 & 5.001 & -16.723 & -44.961 & -2.391 & 16.163 & -47.740 & -56.396 \\
\hline \multicolumn{9}{|c|}{$\exp -L R_{T}$} \\
\hline$a_{0}$ & 40.067 & 103.672 & 156.828 & 186.745 & 98.406 & 99.830 & 72.113 & 11.858 \\
\hline$a_{-1}$ & -36.364 & -99.066 & -151.349 & -180.362 & -94.030 & -94.459 & -65.837 & -4.608 \\
\hline$a_{1}$ & -36.349 & -97.902 & -149.611 & -179.484 & -93.976 & -93.386 & -64.653 & -5.442 \\
\hline$a_{2}$ & 32.965 & 85.377 & 131.562 & 163.393 & 87.219 & 81.303 & 54.872 & 8.838 \\
\hline$a_{3}$ & -19.352 & -45.650 & -71.917 & -95.791 & -52.039 & -43.824 & -28.958 & -11.955 \\
\hline
\end{tabular}

Table B.5: Coefficients of the response surface regressions (Model II-b)

\begin{tabular}{|c|c|c|c|c|c|c|c|c|}
\hline & \multicolumn{2}{|c|}{ confidence level $=0.9$} & \multicolumn{6}{|c|}{ confidence level $=0.95$} \\
\hline & $p_{f}^{z}=1$ & 2 & 3 & 4 & $p_{f}^{z}=1$ & 2 & 3 & 4 \\
\hline \multicolumn{9}{|c|}{ sup- $L R_{T}$} \\
\hline$a_{0}$ & 328.702 & 334.786 & 346.856 & 257.698 & 362.135 & 396.644 & 311.699 & 452.329 \\
\hline$a_{-1}$ & -315.765 & -321.771 & -333.813 & -244.557 & -347.580 & -381.994 & -297.061 & -437.608 \\
\hline$a_{1}$ & -313.389 & -318.486 & -329.606 & -241.258 & -342.930 & -377.776 & -292.785 & -431.854 \\
\hline$a_{2}$ & 287.581 & 288.122 & 292.281 & 212.770 & 302.797 & 336.200 & 256.617 & 381.415 \\
\hline$a_{3}$ & -180.414 & -175.235 & -167.344 & -121.852 & -173.933 & -192.287 & -143.199 & -212.898 \\
\hline \multicolumn{9}{|c|}{ avg- $L R_{T}$} \\
\hline$a_{0}$ & -47.527 & 26.397 & 16.797 & 15.562 & -33.608 & 20.189 & 90.073 & 107.529 \\
\hline$a_{-1}$ & 50.677 & -23.232 & -13.645 & -12.400 & 37.283 & -16.484 & -86.364 & -103.796 \\
\hline$a_{1}$ & 50.875 & -22.646 & -12.646 & -11.601 & 38.181 & -15.249 & -84.382 & -102.383 \\
\hline$a_{2}$ & -46.729 & 19.992 & 7.879 & 7.609 & -34.755 & 12.295 & 72.716 & 91.846 \\
\hline$a_{3}$ & 30.778 & -7.188 & 3.431 & 2.434 & 24.880 & -0.170 & -31.465 & -45.685 \\
\hline \multicolumn{9}{|c|}{$\exp -L R_{T}$} \\
\hline$a_{0}$ & -42.071 & 23.915 & 76.243 & 132.601 & -128.274 & 26.718 & 82.005 & 96.941 \\
\hline$a_{-1}$ & 44.739 & -21.250 & -73.595 & -129.919 & 131.555 & -23.446 & -78.784 & -93.699 \\
\hline$a_{1}$ & 44.922 & -20.306 & -71.722 & -128.177 & 131.258 & -22.277 & -76.734 & -91.967 \\
\hline$a_{2}$ & -42.682 & 15.507 & 58.774 & 111.877 & -121.773 & 15.717 & 63.289 & 78.577 \\
\hline$a_{3}$ & 25.522 & -7.076 & -27.017 & -58.853 & 72.197 & -3.896 & -28.854 & -38.897 \\
\hline
\end{tabular}


Table B.6: Coefficients of the response surface regressions (Model II-c)

\begin{tabular}{|c|c|c|c|c|c|c|c|}
\hline & & \multicolumn{2}{|c|}{ confidence level $=0.9$} & \multicolumn{4}{|c|}{ confidence level $=0.95$} \\
\hline & & $p_{f}^{z}=1$ & 2 & 3 & $p_{f}^{z}=1$ & 2 & 3 \\
\hline \multirow[t]{18}{*}{$p_{b}^{z}=1$} & \multicolumn{7}{|c|}{$\sup -L R_{T}$} \\
\hline & $a_{0}$ & 335.274 & 348.389 & 451.206 & 128.014 & 524.278 & 144.918 \\
\hline & $a_{-1}$ & -320.020 & -333.177 & -435.934 & -111.019 & -507.412 & -127.889 \\
\hline & $a_{1}$ & -318.772 & -331.286 & -433.120 & -110.203 & -502.047 & -127.067 \\
\hline & $a_{2}$ & 297.613 & 305.824 & 397.958 & 103.123 & 449.871 & 119.633 \\
\hline & $a_{3}$ & -192.373 & -190.989 & -243.560 & -75.545 & -259.791 & -85.093 \\
\hline & \multicolumn{7}{|c|}{$\operatorname{avg}-L R_{T}$} \\
\hline & $a_{0}$ & 48.859 & 77.250 & 5.872 & 6.978 & -48.210 & 37.485 \\
\hline & $a_{-1}$ & -44.417 & -72.837 & -1.431 & -1.886 & 53.271 & -32.442 \\
\hline & $a_{1}$ & -43.858 & -71.725 & -1.230 & -1.829 & 53.587 & -31.341 \\
\hline & $a_{2}$ & 41.912 & 65.283 & 2.668 & 6.678 & -46.686 & 29.331 \\
\hline & $a_{3}$ & -21.831 & -32.141 & 1.858 & -1.975 & 32.086 & -9.949 \\
\hline & \multicolumn{7}{|c|}{$\exp -L R_{T}$} \\
\hline & $a_{0}$ & 35.927 & 32.331 & 11.703 & 110.311 & 126.707 & 126.436 \\
\hline & $a_{-1}$ & -32.253 & -28.693 & -8.058 & -105.973 & -122.412 & -122.153 \\
\hline & $a_{1}$ & -31.709 & -27.888 & -7.561 & -104.863 & -120.880 & -120.698 \\
\hline & $a_{2}$ & 27.280 & 22.968 & 5.399 & 94.251 & 106.734 & 108.314 \\
\hline & $a_{3}$ & -14.855 & -11.444 & -2.367 & -53.322 & -56.984 & -60.677 \\
\hline \multirow[t]{18}{*}{$p_{b}^{z}=2$} & \multicolumn{7}{|c|}{ sup- $L R_{T}$} \\
\hline & $a_{0}$ & 468.094 & 205.131 & & 211.642 & 45.048 & \\
\hline & $a_{-1}$ & -450.681 & -187.666 & & -192.428 & -25.757 & \\
\hline & $a_{1}$ & -447.824 & -186.945 & & -191.186 & -25.702 & \\
\hline & $a_{2}$ & 405.279 & 170.669 & & 174.401 & 24.190 & \\
\hline & $a_{3}$ & -240.676 & -109.431 & & -112.630 & -27.104 & \\
\hline & \multicolumn{7}{|c|}{$\operatorname{avg}-L R_{T}$} \\
\hline & $a_{0}$ & 51.807 & 75.889 & & 147.598 & 47.473 & \\
\hline & $a_{-1}$ & -46.117 & -70.191 & & -141.296 & -41.090 & \\
\hline & $a_{1}$ & -45.555 & -69.196 & & -138.779 & -40.375 & \\
\hline & $a_{2}$ & 44.600 & 63.949 & & 125.323 & 41.374 & \\
\hline & $a_{3}$ & -24.737 & -32.509 & & -62.270 & -21.834 & \\
\hline & \multicolumn{7}{|c|}{$\exp -L R_{T}$} \\
\hline & $a_{0}$ & 73.477 & 44.888 & & 119.248 & 121.963 & \\
\hline & $a_{-1}$ & -68.866 & -40.253 & & -113.902 & -116.606 & \\
\hline & $a_{1}$ & -68.134 & -39.931 & & -112.207 & -115.649 & \\
\hline & $a_{2}$ & 60.564 & 35.440 & & 96.830 & 105.056 & \\
\hline & $a_{3}$ & -34.944 & -20.800 & & -50.816 & -62.001 & \\
\hline \multirow[t]{18}{*}{$p_{b}^{z}=3$} & \multicolumn{7}{|c|}{ sup- $L R_{T}$} \\
\hline & $a_{0}$ & 385.532 & & & 335.216 & & \\
\hline & $a_{-1}$ & -366.242 & & & -314.067 & & \\
\hline & $a_{1}$ & -364.027 & & & -311.014 & & \\
\hline & $a_{2}$ & 333.115 & & & 279.456 & & \\
\hline & $a_{3}$ & -206.430 & & & -169.314 & & \\
\hline & \multicolumn{7}{|c|}{$\operatorname{avg}-L R_{T}$} \\
\hline & $a_{0}$ & 64.721 & & & -1.855 & & \\
\hline & $a_{-1}$ & -57.822 & & & 9.514 & & \\
\hline & $a_{1}$ & -57.221 & & & 9.304 & & \\
\hline & $a_{2}$ & 55.161 & & & -0.485 & & \\
\hline & $a_{3}$ & -28.746 & & & 0.244 & & \\
\hline & \multicolumn{7}{|c|}{$\exp -L R_{T}$} \\
\hline & $a_{0}$ & 132.675 & & & 68.744 & & \\
\hline & $a_{-1}$ & -127.162 & & & -62.447 & & \\
\hline & $a_{1}$ & -126.196 & & & -61.845 & & \\
\hline & $a_{2}$ & 113.289 & & & 55.648 & & \\
\hline & $a_{3}$ & -64.996 & & & -33.249 & & \\
\hline
\end{tabular}


Table 1: Coverage rates and lengths of the confidence sets (Model I-a)

\begin{tabular}{|c|c|c|c|c|c|c|c|c|}
\hline \multirow[b]{2}{*}{$\mathrm{d}$} & \multicolumn{4}{|c|}{ Coverage rates } & \multicolumn{4}{|c|}{ Lengths of the confidence sets } \\
\hline & BLS & sup & avg & $\exp$ & BLS & sup & avg & $\exp$ \\
\hline \multicolumn{2}{|c|}{ DGP1 } & & & $T=100$ & & & & \\
\hline 4 & 0.789 & 0.937 & 0.908 & 0.897 & 0.178 & 0.303 & 0.273 & 0.259 \\
\hline 8 & 0.901 & 0.953 & 0.927 & 0.917 & 0.108 & 0.141 & 0.131 & 0.120 \\
\hline 12 & 0.942 & 0.960 & 0.934 & 0.925 & 0.083 & 0.086 & 0.084 & 0.074 \\
\hline 16 & 0.963 & 0.963 & 0.938 & 0.928 & 0.072 & 0.061 & 0.063 & 0.053 \\
\hline \multicolumn{9}{|c|}{ DGP2 } \\
\hline 4 & 0.807 & 0.941 & 0.908 & 0.904 & 0.153 & 0.248 & 0.220 & 0.209 \\
\hline 8 & 0.910 & 0.958 & 0.924 & 0.925 & 0.108 & 0.108 & 0.102 & 0.092 \\
\hline 12 & 0.952 & 0.965 & 0.930 & 0.933 & 0.088 & 0.066 & 0.067 & 0.057 \\
\hline 16 & 0.968 & 0.967 & 0.932 & 0.936 & 0.077 & 0.048 & 0.052 & 0.042 \\
\hline \multicolumn{9}{|c|}{ DGP3 } \\
\hline 4 & 0.645 & 0.877 & 0.792 & 0.807 & 0.241 & 0.420 & 0.352 & 0.347 \\
\hline 8 & 0.811 & 0.932 & 0.867 & 0.883 & 0.153 & 0.227 & 0.192 & 0.186 \\
\hline 12 & 0.891 & 0.956 & 0.902 & 0.916 & 0.116 & 0.148 & 0.127 & 0.122 \\
\hline 16 & 0.924 & 0.968 & 0.917 & 0.932 & 0.100 & 0.107 & 0.094 & 0.088 \\
\hline \multicolumn{9}{|c|}{ DGP4 } \\
\hline 4 & 0.808 & 0.938 & 0.907 & 0.902 & 0.156 & 0.260 & 0.237 & 0.222 \\
\hline 8 & 0.926 & 0.955 & 0.926 & 0.924 & 0.098 & 0.120 & 0.115 & 0.102 \\
\hline 12 & 0.960 & 0.961 & 0.932 & 0.930 & 0.077 & 0.074 & 0.077 & 0.064 \\
\hline 16 & 0.972 & 0.962 & 0.934 & 0.932 & 0.066 & 0.053 & 0.060 & 0.046 \\
\hline \multicolumn{9}{|c|}{ DGP5 } \\
\hline 4 & 0.691 & 0.859 & 0.750 & 0.774 & 0.175 & 0.285 & 0.229 & 0.227 \\
\hline 8 & 0.847 & 0.921 & 0.827 & 0.853 & 0.126 & 0.147 & 0.121 & 0.116 \\
\hline 12 & 0.917 & 0.949 & 0.861 & 0.886 & 0.106 & 0.094 & 0.082 & 0.076 \\
\hline 16 & 0.952 & 0.960 & 0.879 & 0.904 & 0.093 & 0.069 & 0.064 & 0.056 \\
\hline \multicolumn{2}{|c|}{ 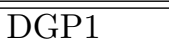 } & & & $T=300$ & & & & \\
\hline 4 & 0.833 & 0.953 & 0.940 & 0.940 & 0.126 & 0.234 & 0.225 & 0.213 \\
\hline 8 & 0.918 & 0.959 & 0.944 & 0.944 & 0.072 & 0.100 & 0.103 & 0.092 \\
\hline 12 & 0.954 & 0.960 & 0.945 & 0.946 & 0.051 & 0.060 & 0.064 & 0.055 \\
\hline 16 & 0.963 & 0.962 & 0.946 & 0.948 & 0.043 & 0.041 & 0.046 & 0.039 \\
\hline \multicolumn{9}{|c|}{ DGP2 } \\
\hline 4 & 0.852 & 0.952 & 0.936 & 0.939 & 0.117 & 0.181 & 0.174 & 0.163 \\
\hline 8 & 0.925 & 0.957 & 0.940 & 0.943 & 0.069 & 0.071 & 0.076 & 0.066 \\
\hline 12 & 0.954 & 0.958 & 0.942 & 0.945 & 0.051 & 0.043 & 0.050 & 0.041 \\
\hline 16 & 0.971 & 0.959 & 0.942 & 0.945 & 0.043 & 0.031 & 0.037 & 0.030 \\
\hline \multicolumn{9}{|c|}{ DGP3 } \\
\hline 4 & 0.686 & 0.928 & 0.885 & 0.892 & 0.204 & 0.404 & 0.361 & 0.354 \\
\hline 8 & 0.833 & 0.950 & 0.911 & 0.918 & 0.120 & 0.198 & 0.180 & 0.171 \\
\hline 12 & 0.900 & 0.959 & 0.922 & 0.929 & 0.088 & 0.122 & 0.115 & 0.107 \\
\hline 16 & 0.932 & 0.965 & 0.929 & 0.935 & 0.071 & 0.086 & 0.083 & 0.076 \\
\hline \multicolumn{9}{|c|}{ DGP4 } \\
\hline 4 & 0.848 & 0.953 & 0.938 & 0.937 & 0.112 & 0.196 & 0.188 & 0.177 \\
\hline 8 & 0.935 & 0.958 & 0.942 & 0.943 & 0.066 & 0.082 & 0.086 & 0.075 \\
\hline 12 & 0.956 & 0.960 & 0.943 & 0.945 & 0.047 & 0.049 & 0.054 & 0.046 \\
\hline 16 & 0.968 & 0.961 & 0.944 & 0.947 & 0.038 & 0.034 & 0.040 & 0.033 \\
\hline \multicolumn{9}{|c|}{ DGP5 } \\
\hline 4 & 0.756 & 0.926 & 0.874 & 0.884 & 0.164 & 0.276 & 0.240 & 0.235 \\
\hline 8 & 0.889 & 0.949 & 0.901 & 0.916 & 0.106 & 0.123 & 0.112 & 0.105 \\
\hline 12 & 0.931 & 0.958 & 0.909 & 0.925 & 0.082 & 0.074 & 0.071 & 0.065 \\
\hline 16 & 0.949 & 0.962 & 0.915 & 0.929 & 0.066 & 0.052 & 0.052 & 0.046 \\
\hline
\end{tabular}


Table 2: Coverage rates and lengths of the confidence sets (Model I-b)

\begin{tabular}{|c|c|c|c|c|c|c|c|c|}
\hline \multirow[b]{2}{*}{$\mathrm{d}$} & \multicolumn{4}{|c|}{ Coverage rates } & \multicolumn{4}{|c|}{ Lengths of the confidence sets } \\
\hline & BLS & sup & avg & $\exp$ & BLS & sup & avg & $\exp$ \\
\hline \multicolumn{2}{|c|}{ DGP1 } & & & $T=100$ & & & & \\
\hline 4 & 0.886 & 0.952 & 0.934 & 0.934 & 0.151 & 0.216 & 0.229 & 0.189 \\
\hline 8 & 0.978 & 0.965 & 0.947 & 0.947 & 0.053 & 0.058 & 0.075 & 0.055 \\
\hline 12 & 0.996 & 0.969 & 0.949 & 0.951 & 0.031 & 0.038 & 0.046 & 0.036 \\
\hline 16 & 1.000 & 0.970 & 0.950 & 0.953 & 0.030 & 0.028 & 0.035 & 0.027 \\
\hline \multicolumn{9}{|c|}{ DGP2 } \\
\hline 4 & 0.875 & 0.950 & 0.930 & 0.929 & 0.150 & 0.226 & 0.239 & 0.198 \\
\hline 8 & 0.975 & 0.965 & 0.945 & 0.945 & 0.053 & 0.059 & 0.077 & 0.056 \\
\hline 12 & 0.996 & 0.968 & 0.948 & 0.950 & 0.031 & 0.038 & 0.047 & 0.036 \\
\hline 16 & 1.000 & 0.970 & 0.949 & 0.951 & 0.030 & 0.028 & 0.035 & 0.027 \\
\hline \multicolumn{9}{|c|}{ DGP3 } \\
\hline 4 & 0.687 & 0.870 & 0.810 & 0.806 & 0.283 & 0.421 & 0.361 & 0.349 \\
\hline 8 & 0.882 & 0.928 & 0.877 & 0.884 & 0.114 & 0.147 & 0.149 & 0.125 \\
\hline 12 & 0.964 & 0.963 & 0.914 & 0.925 & 0.062 & 0.069 & 0.081 & 0.062 \\
\hline 16 & 0.991 & 0.975 & 0.925 & 0.940 & 0.043 & 0.048 & 0.055 & 0.044 \\
\hline \multicolumn{9}{|c|}{ DGP4 } \\
\hline 4 & 0.912 & 0.950 & 0.933 & 0.929 & 0.120 & 0.166 & 0.189 & 0.149 \\
\hline 8 & 0.984 & 0.965 & 0.947 & 0.945 & 0.045 & 0.050 & 0.064 & 0.049 \\
\hline 12 & 0.999 & 0.968 & 0.948 & 0.949 & 0.030 & 0.035 & 0.042 & 0.034 \\
\hline 16 & 1.000 & 0.967 & 0.947 & 0.948 & 0.030 & 0.029 & 0.033 & 0.028 \\
\hline \multicolumn{9}{|c|}{ DGP5 } \\
\hline 4 & 0.666 & 0.838 & 0.794 & 0.776 & 0.221 & 0.352 & 0.309 & 0.288 \\
\hline 8 & 0.890 & 0.921 & 0.869 & 0.873 & 0.086 & 0.115 & 0.123 & 0.098 \\
\hline 12 & 0.973 & 0.960 & 0.903 & 0.917 & 0.049 & 0.056 & 0.066 & 0.051 \\
\hline 16 & 0.996 & 0.969 & 0.914 & 0.928 & 0.035 & 0.042 & 0.047 & 0.038 \\
\hline \multicolumn{2}{|c|}{ 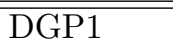 } & & & $T=300$ & & & & \\
\hline 4 & 0.925 & 0.954 & 0.954 & 0.949 & 0.087 & 0.118 & 0.158 & 0.119 \\
\hline 8 & 0.971 & 0.958 & 0.957 & 0.952 & 0.026 & 0.043 & 0.057 & 0.044 \\
\hline 12 & 0.990 & 0.959 & 0.958 & 0.954 & 0.016 & 0.029 & 0.038 & 0.029 \\
\hline 16 & 0.995 & 0.959 & 0.958 & 0.954 & 0.010 & 0.021 & 0.028 & 0.022 \\
\hline \multicolumn{9}{|c|}{ DGP2 } \\
\hline 4 & 0.925 & 0.953 & 0.953 & 0.949 & 0.087 & 0.120 & 0.160 & 0.121 \\
\hline 8 & 0.969 & 0.958 & 0.957 & 0.952 & 0.026 & 0.043 & 0.057 & 0.044 \\
\hline 12 & 0.990 & 0.959 & 0.957 & 0.953 & 0.016 & 0.029 & 0.038 & 0.029 \\
\hline 16 & 0.995 & 0.960 & 0.957 & 0.954 & 0.010 & 0.022 & 0.028 & 0.022 \\
\hline \multicolumn{9}{|c|}{ DGP3 } \\
\hline 4 & 0.776 & 0.924 & 0.901 & 0.898 & 0.236 & 0.380 & 0.351 & 0.332 \\
\hline 8 & 0.918 & 0.948 & 0.923 & 0.924 & 0.074 & 0.100 & 0.125 & 0.095 \\
\hline 12 & 0.961 & 0.958 & 0.933 & 0.937 & 0.036 & 0.053 & 0.068 & 0.052 \\
\hline 16 & 0.985 & 0.962 & 0.937 & 0.941 & 0.023 & 0.039 & 0.049 & 0.039 \\
\hline \multicolumn{9}{|c|}{ DGP4 } \\
\hline 4 & 0.936 & 0.953 & 0.949 & 0.946 & 0.066 & 0.084 & 0.119 & 0.086 \\
\hline 8 & 0.978 & 0.957 & 0.952 & 0.950 & 0.021 & 0.037 & 0.049 & 0.038 \\
\hline 12 & 0.991 & 0.957 & 0.952 & 0.951 & 0.013 & 0.025 & 0.032 & 0.025 \\
\hline 16 & 0.999 & 0.958 & 0.952 & 0.951 & 0.010 & 0.019 & 0.024 & 0.019 \\
\hline \multicolumn{9}{|c|}{ DGP5 } \\
\hline 4 & 0.788 & 0.914 & 0.894 & 0.885 & 0.185 & 0.297 & 0.286 & 0.259 \\
\hline 8 & 0.914 & 0.944 & 0.918 & 0.920 & 0.055 & 0.074 & 0.096 & 0.072 \\
\hline 12 & 0.965 & 0.955 & 0.927 & 0.932 & 0.028 & 0.045 & 0.056 & 0.044 \\
\hline 16 & 0.989 & 0.958 & 0.930 & 0.937 & 0.019 & 0.034 & 0.041 & 0.033 \\
\hline
\end{tabular}


Table 3: Coverage rates and lengths of the confidence sets (Model II-a)

\begin{tabular}{|c|c|c|c|c|c|c|c|c|}
\hline \multirow[b]{2}{*}{$\mathrm{d}$} & \multicolumn{4}{|c|}{ Coverage rates } & \multicolumn{4}{|c|}{ Lengths of the confidence sets } \\
\hline & BLS & sup & avg & $\exp$ & BLS & sup & avg & $\exp$ \\
\hline \multicolumn{2}{|c|}{ DGP1 } & & & $T=100$ & & & & \\
\hline 4 & 0.956 & 0.956 & 0.915 & 0.911 & 0.047 & 0.082 & 0.081 & 0.063 \\
\hline 8 & 0.996 & 0.962 & 0.923 & 0.920 & 0.032 & 0.020 & 0.028 & 0.016 \\
\hline 12 & 0.999 & 0.963 & 0.923 & 0.920 & 0.030 & 0.012 & 0.019 & 0.011 \\
\hline 16 & 1.000 & 0.963 & 0.923 & 0.920 & 0.030 & 0.010 & 0.015 & 0.010 \\
\hline \multicolumn{9}{|c|}{ DGP2 } \\
\hline 4 & 0.879 & 0.943 & 0.892 & 0.894 & 0.061 & 0.144 & 0.130 & 0.114 \\
\hline 8 & 0.960 & 0.956 & 0.911 & 0.911 & 0.045 & 0.050 & 0.050 & 0.040 \\
\hline 12 & 0.979 & 0.958 & 0.916 & 0.915 & 0.041 & 0.028 & 0.030 & 0.023 \\
\hline 16 & 0.990 & 0.959 & 0.917 & 0.916 & 0.039 & 0.020 & 0.023 & 0.018 \\
\hline \multicolumn{9}{|c|}{ DGP3 } \\
\hline 4 & 0.815 & 0.923 & 0.831 & 0.856 & 0.080 & 0.193 & 0.155 & 0.141 \\
\hline 8 & 0.982 & 0.983 & 0.925 & 0.938 & 0.038 & 0.047 & 0.048 & 0.035 \\
\hline 12 & 0.996 & 0.987 & 0.933 & 0.945 & 0.032 & 0.021 & 0.028 & 0.017 \\
\hline 16 & 0.998 & 0.988 & 0.934 & 0.946 & 0.031 & 0.014 & 0.021 & 0.012 \\
\hline \multicolumn{9}{|c|}{ DGP4 } \\
\hline 4 & 0.968 & 0.949 & 0.904 & 0.899 & 0.040 & 0.064 & 0.070 & 0.049 \\
\hline 8 & 0.997 & 0.954 & 0.909 & 0.905 & 0.031 & 0.017 & 0.028 & 0.014 \\
\hline 12 & 0.999 & 0.954 & 0.909 & 0.905 & 0.030 & 0.011 & 0.019 & 0.010 \\
\hline 16 & 1.000 & 0.954 & 0.910 & 0.906 & 0.030 & 0.010 & 0.015 & 0.009 \\
\hline \multicolumn{9}{|c|}{ DGP5 } \\
\hline 4 & 0.772 & 0.869 & 0.737 & 0.767 & 0.070 & 0.168 & 0.129 & 0.119 \\
\hline 8 & 0.931 & 0.942 & 0.819 & 0.853 & 0.049 & 0.068 & 0.057 & 0.048 \\
\hline 12 & 0.966 & 0.957 & 0.837 & 0.871 & 0.044 & 0.038 & 0.035 & 0.028 \\
\hline 16 & 0.981 & 0.962 & 0.845 & 0.878 & 0.042 & 0.027 & 0.026 & 0.021 \\
\hline \multicolumn{2}{|c|}{ 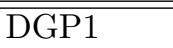 } & & & $T=300$ & & & & \\
\hline 4 & 0.967 & 0.962 & 0.939 & 0.948 & 0.024 & 0.044 & 0.051 & 0.039 \\
\hline 8 & 0.995 & 0.963 & 0.941 & 0.950 & 0.012 & 0.014 & 0.018 & 0.013 \\
\hline 12 & 0.998 & 0.963 & 0.941 & 0.950 & 0.011 & 0.008 & 0.012 & 0.008 \\
\hline 16 & 1.000 & 0.963 & 0.941 & 0.950 & 0.010 & 0.006 & 0.010 & 0.006 \\
\hline \multicolumn{9}{|c|}{ DGP2 } \\
\hline 4 & 0.910 & 0.959 & 0.936 & 0.941 & 0.038 & 0.098 & 0.096 & 0.086 \\
\hline 8 & 0.968 & 0.962 & 0.939 & 0.945 & 0.024 & 0.033 & 0.035 & 0.029 \\
\hline 12 & 0.983 & 0.963 & 0.939 & 0.945 & 0.020 & 0.018 & 0.021 & 0.017 \\
\hline 16 & 0.988 & 0.963 & 0.939 & 0.946 & 0.017 & 0.012 & 0.016 & 0.012 \\
\hline \multicolumn{9}{|c|}{ DGP3 } \\
\hline 4 & 0.855 & 0.950 & 0.899 & 0.915 & 0.056 & 0.168 & 0.151 & 0.133 \\
\hline 8 & 0.975 & 0.974 & 0.927 & 0.944 & 0.020 & 0.034 & 0.035 & 0.029 \\
\hline 12 & 0.995 & 0.977 & 0.931 & 0.947 & 0.013 & 0.018 & 0.020 & 0.016 \\
\hline 16 & 0.997 & 0.977 & 0.931 & 0.947 & 0.011 & 0.012 & 0.015 & 0.011 \\
\hline \multicolumn{9}{|c|}{ DGP4 } \\
\hline 4 & 0.979 & 0.962 & 0.939 & 0.943 & 0.020 & 0.035 & 0.040 & 0.031 \\
\hline 8 & 0.997 & 0.962 & 0.939 & 0.944 & 0.011 & 0.012 & 0.017 & 0.011 \\
\hline 12 & 0.999 & 0.962 & 0.939 & 0.944 & 0.010 & 0.007 & 0.012 & 0.006 \\
\hline 16 & 0.999 & 0.962 & 0.940 & 0.944 & 0.010 & 0.005 & 0.009 & 0.005 \\
\hline \multicolumn{9}{|c|}{ DGP5 } \\
\hline 4 & 0.815 & 0.934 & 0.865 & 0.885 & 0.055 & 0.166 & 0.142 & 0.136 \\
\hline 8 & 0.934 & 0.959 & 0.898 & 0.916 & 0.032 & 0.060 & 0.054 & 0.049 \\
\hline 12 & 0.971 & 0.966 & 0.908 & 0.926 & 0.024 & 0.032 & 0.031 & 0.027 \\
\hline 16 & 0.980 & 0.967 & 0.910 & 0.929 & 0.022 & 0.021 & 0.022 & 0.018 \\
\hline
\end{tabular}


Table 4: Coverage rates and lengths of the confidence sets (Model II-b)

\begin{tabular}{|c|c|c|c|c|c|c|c|c|}
\hline \multirow[b]{2}{*}{$\mathrm{d}$} & \multicolumn{4}{|c|}{ Coverage rates } & \multicolumn{4}{|c|}{ Lengths of the confidence sets } \\
\hline & BLS & sup & avg & $\exp$ & BLS & sup & avg & $\exp$ \\
\hline \multicolumn{2}{|c|}{ DGP1 } & & & $T=100$ & & & & \\
\hline 4 & 0.987 & 0.975 & 0.927 & 0.935 & 0.040 & 0.041 & 0.049 & 0.034 \\
\hline 8 & 1.000 & 0.978 & 0.930 & 0.939 & 0.030 & 0.016 & 0.022 & 0.014 \\
\hline 12 & 1.000 & 0.978 & 0.930 & 0.939 & 0.030 & 0.010 & 0.016 & 0.010 \\
\hline 16 & 1.000 & 0.978 & 0.930 & 0.939 & 0.030 & 0.010 & 0.012 & 0.009 \\
\hline \multicolumn{9}{|c|}{ DGP2 } \\
\hline 4 & 0.986 & 0.973 & 0.922 & 0.931 & 0.040 & 0.042 & 0.053 & 0.035 \\
\hline 8 & 1.000 & 0.976 & 0.924 & 0.935 & 0.030 & 0.016 & 0.022 & 0.014 \\
\hline 12 & 1.000 & 0.976 & 0.924 & 0.935 & 0.030 & 0.010 & 0.016 & 0.010 \\
\hline 16 & 1.000 & 0.976 & 0.924 & 0.935 & 0.030 & 0.010 & 0.012 & 0.009 \\
\hline \multicolumn{9}{|c|}{ DGP3 } \\
\hline 4 & 0.866 & 0.925 & 0.815 & 0.847 & 0.073 & 0.157 & 0.128 & 0.104 \\
\hline 8 & 0.997 & 0.983 & 0.885 & 0.923 & 0.032 & 0.029 & 0.028 & 0.023 \\
\hline 12 & 1.000 & 0.984 & 0.887 & 0.923 & 0.030 & 0.016 & 0.020 & 0.013 \\
\hline 16 & 1.000 & 0.984 & 0.887 & 0.923 & 0.030 & 0.011 & 0.015 & 0.010 \\
\hline \multicolumn{9}{|c|}{ DGP4 } \\
\hline 4 & 0.991 & 0.970 & 0.925 & 0.930 & 0.033 & 0.036 & 0.044 & 0.029 \\
\hline 8 & 1.000 & 0.970 & 0.926 & 0.932 & 0.030 & 0.013 & 0.022 & 0.011 \\
\hline 12 & 1.000 & 0.970 & 0.926 & 0.932 & 0.030 & 0.010 & 0.014 & 0.009 \\
\hline 16 & 1.000 & 0.970 & 0.926 & 0.932 & 0.030 & 0.010 & 0.011 & 0.009 \\
\hline \multicolumn{9}{|c|}{ DGP5 } \\
\hline 4 & 0.885 & 0.917 & 0.786 & 0.823 & 0.059 & 0.113 & 0.096 & 0.076 \\
\hline 8 & 1.000 & 0.975 & 0.837 & 0.880 & 0.030 & 0.025 & 0.028 & 0.019 \\
\hline 12 & 1.000 & 0.975 & 0.837 & 0.880 & 0.030 & 0.012 & 0.018 & 0.010 \\
\hline 16 & 1.000 & 0.975 & 0.837 & 0.880 & 0.030 & 0.010 & 0.013 & 0.009 \\
\hline \multicolumn{2}{|c|}{ 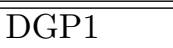 } & & & $T=300$ & & & & \\
\hline 4 & 0.979 & 0.960 & 0.940 & 0.945 & 0.019 & 0.024 & 0.030 & 0.022 \\
\hline 8 & 0.999 & 0.962 & 0.942 & 0.947 & 0.010 & 0.011 & 0.013 & 0.010 \\
\hline 12 & 1.000 & 0.962 & 0.942 & 0.947 & 0.010 & 0.008 & 0.009 & 0.007 \\
\hline 16 & 1.000 & 0.962 & 0.942 & 0.947 & 0.010 & 0.005 & 0.008 & 0.005 \\
\hline \multicolumn{9}{|c|}{ DGP2 } \\
\hline 4 & 0.978 & 0.960 & 0.939 & 0.944 & 0.019 & 0.024 & 0.031 & 0.022 \\
\hline 8 & 0.999 & 0.961 & 0.941 & 0.945 & 0.010 & 0.011 & 0.013 & 0.010 \\
\hline 12 & 1.000 & 0.961 & 0.941 & 0.946 & 0.010 & 0.008 & 0.009 & 0.007 \\
\hline 16 & 1.000 & 0.961 & 0.941 & 0.946 & 0.010 & 0.005 & 0.008 & 0.005 \\
\hline \multicolumn{9}{|c|}{ DGP3 } \\
\hline 4 & 0.901 & 0.943 & 0.889 & 0.902 & 0.049 & 0.108 & 0.111 & 0.081 \\
\hline 8 & 0.994 & 0.965 & 0.911 & 0.927 & 0.017 & 0.021 & 0.023 & 0.018 \\
\hline 12 & 1.000 & 0.965 & 0.913 & 0.928 & 0.010 & 0.013 & 0.014 & 0.012 \\
\hline 16 & 1.000 & 0.965 & 0.913 & 0.928 & 0.010 & 0.010 & 0.011 & 0.009 \\
\hline \multicolumn{9}{|c|}{ DGP4 } \\
\hline 4 & 0.989 & 0.960 & 0.939 & 0.942 & 0.016 & 0.020 & 0.025 & 0.019 \\
\hline 8 & 1.000 & 0.960 & 0.940 & 0.942 & 0.010 & 0.010 & 0.013 & 0.010 \\
\hline 12 & 1.000 & 0.960 & 0.940 & 0.942 & 0.010 & 0.007 & 0.010 & 0.006 \\
\hline 16 & 1.000 & 0.960 & 0.940 & 0.942 & 0.010 & 0.004 & 0.008 & 0.004 \\
\hline \multicolumn{9}{|c|}{ DGP5 } \\
\hline 4 & 0.919 & 0.946 & 0.886 & 0.901 & 0.037 & 0.067 & 0.076 & 0.052 \\
\hline 8 & 0.996 & 0.965 & 0.905 & 0.922 & 0.014 & 0.018 & 0.020 & 0.016 \\
\hline 12 & 1.000 & 0.965 & 0.904 & 0.922 & 0.010 & 0.012 & 0.014 & 0.011 \\
\hline 16 & 1.000 & 0.965 & 0.905 & 0.922 & 0.010 & 0.009 & 0.012 & 0.008 \\
\hline
\end{tabular}


Table 5: Empirical results

\begin{tabular}{lll}
\hline \hline series: $y_{t}, p_{t}^{\text {oil }}$ & & \\
\hline estimated break date & $:$ & $2008 \mathrm{Q} 3$ \\
cointegration test with a break & $:$ & 0.057 \\
cointegration test without a break & $:$ & $0.172^{* *}$ \\
LM test for a break & $:$ & $11.322^{* * *}$ \\
Wald test for a break & $:$ & $401.180^{* * *}$ \\
& & \\
series: $c_{t}, p_{t}^{\text {oil }}$ & $:$ & $2008 \mathrm{Q} 4$ \\
\hline estimated break date & $:$ & 0.048 \\
cointegration test with a break & $:$ & $0.117^{*}$ \\
cointegration test without a break & $:$ & $12.134^{* * *}$ \\
LM test for a break & $:$ & $195.130^{* * *}$ \\
Wald test for a break & \\
& $:$ & \\
series: $i_{t}, p_{t}^{\text {oil }}$ & $:$ & 0.049 \\
\hline estimated break date & $:$ & $0.131^{* *}$ \\
cointegration test with a break & $:$ & $10.808^{* * *}$ \\
cointegration test without a break & $:$ & $101.683^{* * *}$ \\
LM test for a break & &
\end{tabular}

Note: the superscripts $*, * *$, and ${ }^{* * *}$ signify significance at $10 \%, 5 \%$ and $1 \%$, respectively. 


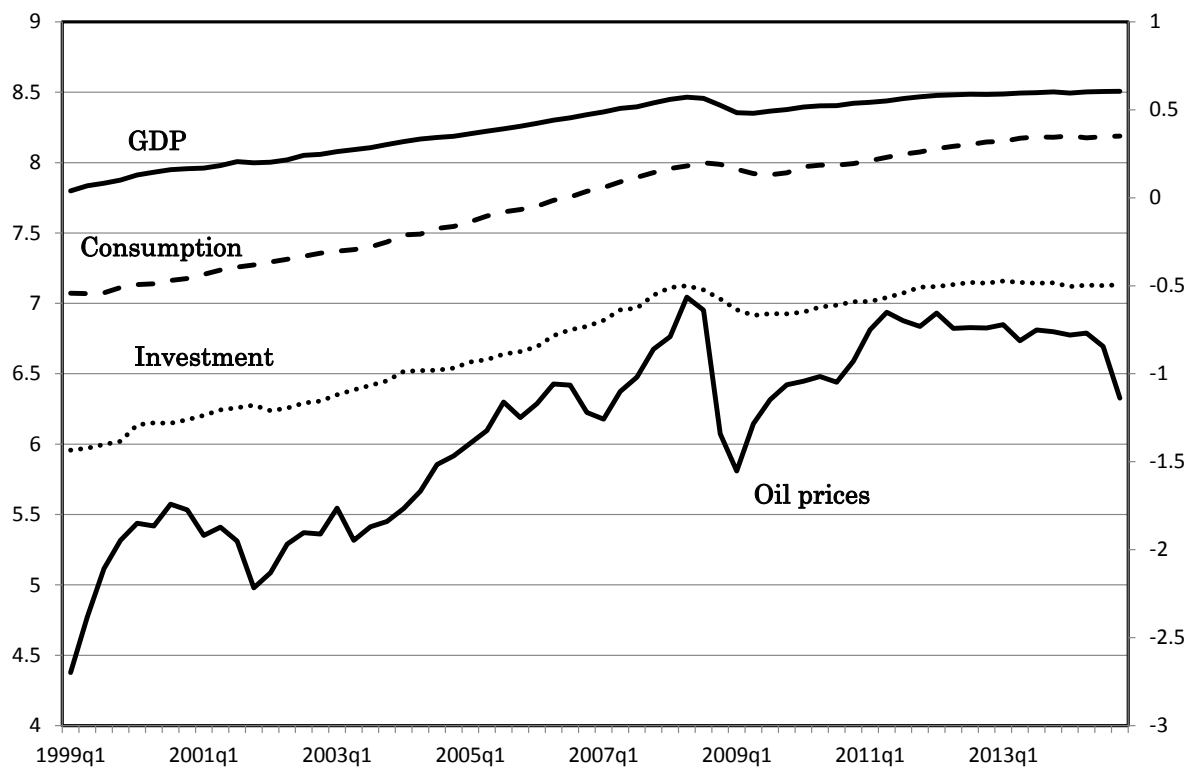

Figure 1: Russian macroeconomic series and oil prices 


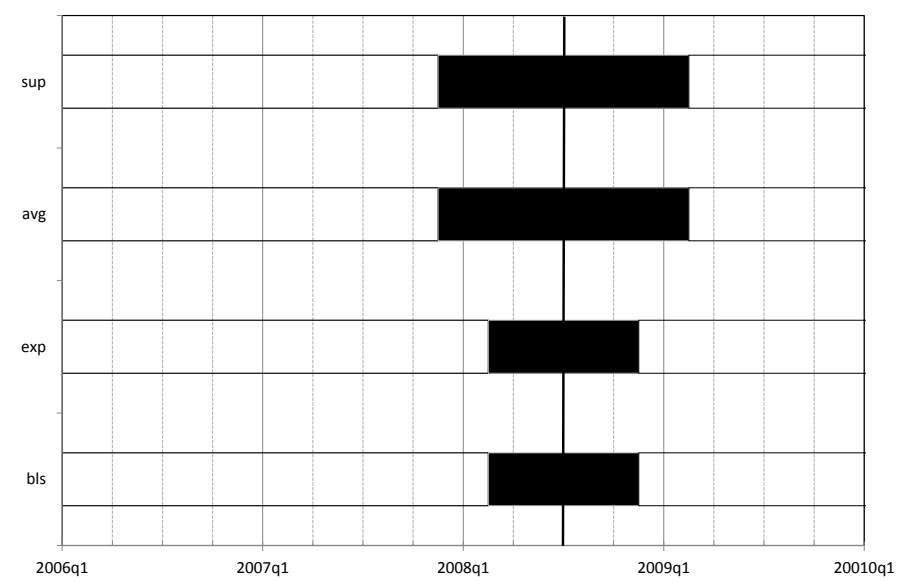

(i) $y_{t}$

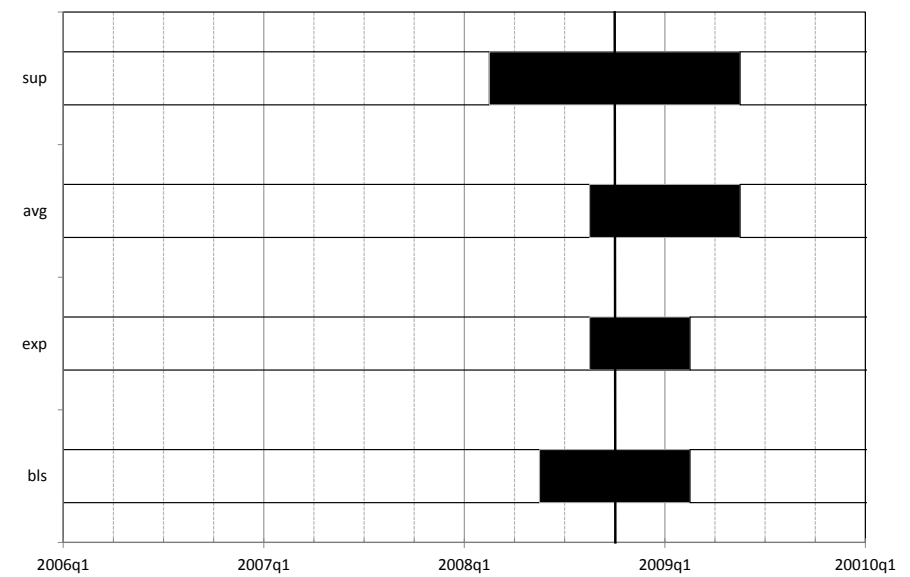

(ii) $c_{t}$

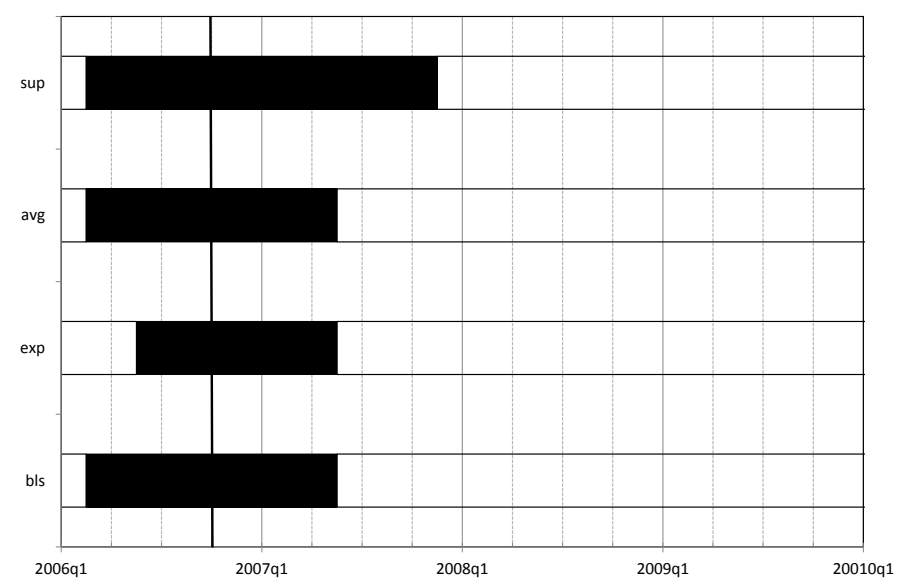

(iii) $i_{t}$

Figure 2: Confidence sets for a break 